\title{
New insights on the interfacial tension of electrochemical interfaces and the Lippmann equation
}

\author{
W. DREYER, C. GUHLKE, M. LANDSTORFER and R. MÜLLER \\ Weierstrass-Institute, Mohrenstr. 39, 10117 Berlin, Germany \\ emails:Wolfgang.Dreyer@wias-berlin.de,Clemens.Guhlke@wias-berlin.de,Manuel.Landstrofer@wias-berlin.de, \\ Ruediger.Mueller@wias-berlin.de
}

(Received 8 June 2017; revised 14 November 2017; accepted 14 November 2017; first published online 13 December 2017)

\begin{abstract}
The Lippmann equation is considered as universal relationship between interfacial tension, double layer charge, and cell potential. Based on the framework of continuum thermoelectrodynamics, we provide some crucial new insights to this relation. For general interfaces such that the local curvature radius is large compared to the Debye length, we apply asymptotic analysis methods to obtain the Lippmann equation. We give precise definitions of the involved quantities and show that the interfacial tension of the Lippmann equation is composed of the surface tension of our general model, and contributions arising from the adjacent space charge layers that can only lower the interfacial tension. Moreover, it turns out that surface reactions can be consistently incorporated into the Lippmann equation, provided that there is no charge transfer from one side of the interface to the other. We apply the model to curved liquid metal electrodes and compare our model to experimental data of several mercury-electrolyte interfaces. We obtain qualitative and quantitative agreement in the $2 \mathrm{~V}$ potential range for various salt concentrations.
\end{abstract}

Key words: Lippmann equation, electrochemistry, liquid-liquid interface, asymptotic analysis

\section{Introduction}

The interfacial phenomena of electrocapillarity, discovered by Lippmann a century ago $[32,33]$, is a key feature for investigations of the electric double layer which forms at the interface between two charged phases. Intensive experimental studies on mercury-aqueous electrolyte interfaces carried out by Gouy [22-25], Frumkin [18], Grahame [27], and others, lead to the fundamental perceptions of the double layer by Grahame [27]. Experimentally well and reproducible observed is the parabola shaped relationship between the interfacial tension $\gamma$ and some applied voltage $U$, cf. Figure 1a. Moreover, the slope of the surface tension with respect to the applied voltage is given by the double layer charge density $Q$. This relation is known as the Lippmann equation [3,8,37]:

$$
\frac{d}{d U} \gamma=-Q
$$

The thermodynamical basis of this relation is the Gibbs adsorption equation, and a derivation can be found in [27,37]. For experimental verification of the Lippmann equation 
the Young-Laplace equation is used, which relates the pressure difference $p^{+}-p^{-}$between the mercury electrode and the electrolyte to the interfacial tension and the mean curvature of the mercury surface $k_{M}$, viz.

$$
p^{+}-p^{-}=2 k_{M} \gamma
$$

If the mean curvature does not change - which seems to be well satisfied in Lippmann's electrocapillarity experiments [32] - than the interfacial tension is proportional to the pressure difference. Therefore, the electrocapillarity experiments allow to measure the interfacial tension as a function of the applied voltage. By the use of a second experiment, e.g., the dropping mercury electrode, it is possible to measure directly the double layer charge as a function of the applied voltage. These both independent experiments allow an experimental verification of the Lippmann equation. It is shown that the Lippmann equation is satisfied by various mercury-electrolyte systems [18,27].

However, in the context of non-equilibrium (electro-) thermodynamics [2, 6, 14, 34, 35] there are no corresponding relations to the Lippmann equation (1.1) and the YoungLaplace equation (1.2) in the case of non-zero electromagnetic field. The reason for this seemingly contradictory statement to the derivation of Grahame is that the Gibbs adsorption equation only holds in systems where the bulk phases are homogeneous, whereas in the non-equilibrium thermodynamic setting there are pronounced double layer with strong electric fields and charge accumulation in the vicinity of the surface. Therefore, a derivation of Lippmann and Young-Laplace equations in the context of non-equilibrium electro- thermodynamics is desirable because it incorporates more spatial structure of the double layer into the definition of the interfacial tension and double layer charge density and thereby can lead to a better understanding of the double layer phenomena.

Already in the works of Defay and Sanfeld [13] and Hurwitz and d'Alkaine [29] one can find first attempts to derive a Young-Laplace equation in the framework of nonequilibrium thermodynamics with non-zero electric fields. They figure out the existence of an electric field contributing to the surface tension. Due to the missing framework of non-equilibrium thermodynamics of surfaces, which is firstly introduced several years later by Albano and Bedeaux [2,6], they had to use Gibbs equation for their surface theory and there was no way to derive the Lippmann equation on their framework.

In this work, we discuss the equilibrium relations resulting from non-equilibrium thermodynamics for interfaces between two adjacent charged phases. By using matched asymptotic analysis, we are able to show that the Young-Laplace equation and the Lippmann equation result from quite general thermodynamic relations, which are independent of the considered material. Further on, we are able to give precise definitions of the quantities which appear in the Lippmann and Young-Laplace equation. It turns out that the measurable interfacial tension $\gamma$ actually consists of three contributions, i.e., the surface tension $\gamma$ of the material surface $S$, and two boundary layer contributions $\widetilde{\gamma}^{ \pm}$of the respective phase. These contributions are structurally very different since they arise from volume and surface thermodynamics. Accordingly, the double layer charge density $Q$ is composed of a surface part $q$ and a contribution $\widetilde{q}$ from one of the adjacent layers. Knowledge of these structural decompositions is crucial for a model based understanding 

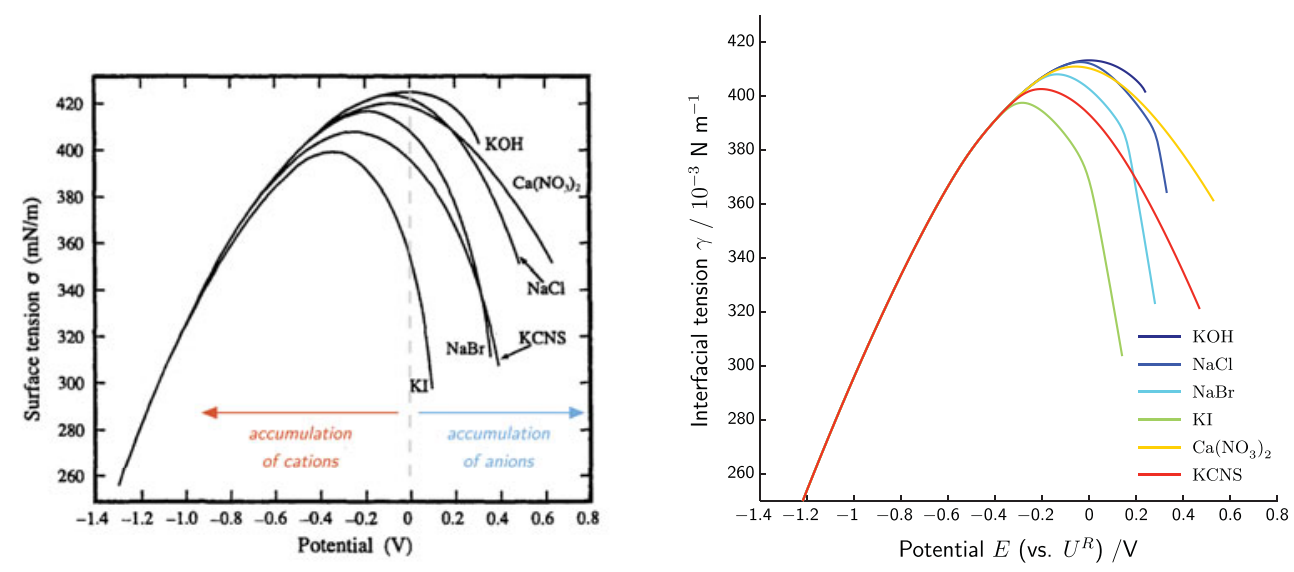

FIGURE 1. Comparison between measured data of electrocapillarity curves and our simulations. (a) Electrocapillarity curves for various salts according to Figure 1 from [27] (reprinted with permission). (b) Computed interfacial tension as described in Section 8.

of the phenomena of electrocapillarity and especially of the electrochemical interfaces itself.

Upon choosing appropriate free energy functions to describe specific material dependent properties of a liquid metal-electrolyte interface, we obtain representations of the interfacial contributions. For example, we get for the electrolytic boundary layer contribution

$$
\widetilde{\gamma}^{-}=\gamma_{\mathrm{BL}}^{\mathrm{E}}=\int_{0}^{U^{\mathrm{E}}} \sqrt{2 \varepsilon_{0}(1+\chi)\left(p\left(U^{\prime}\right)-p^{\mathrm{E}}\right)} d U^{\prime},
$$

where $p^{\mathrm{E}}$ denotes the bulk pressure far away from the interface in the electrolyte, and $p\left(U^{\prime}\right)$ denotes the material pressure at a point in the double layer with a potential difference of $U^{\prime}$ to the bulk electrolyte potential.

For several mercury-aqueous electrolytes we provide numerical computations of the interfacial tension as a function of the applied potential. Figure 1 shows the results in comparison to the well-known measurements by Gouy and Grahame. Moreover, by varying model parameters like the adsorption energies, we can identify mechanisms leading to the deviations of the electrocapillarity curves in Figure 1 for the various salts. Thus, our model allows for a quantitative and qualitative model based understanding of electrocapillarity curves.

\subsection{Outline}

In the next section, we briefly state the complete thermodynamic equilibrium model of two electrochemical systems separated by a curved surface in a material independent form. There is no Lippmann equation at this level but this model is the basis for the following derivation based on asymptotic analysis. Then, we motivate the appearance of additional terms in the Young-Laplace equation in the presence of an electric field 
in in Section 3. In Section 4, we summarize the reduced models for the thin double layer limit and the Lippmann equation with a precise definition of the terms appearing therein. The mathematical derivation of these models and results is then given in Section 5 and Section 6 . Still these results are presented on a material independent level. Next, we validate our model by applying it to various mercury|aqueous electrolyte interfaces. Therefore, we state in Section 7 the material dependent properties that are encoded in the free energy densities. Thereby, we get to a closed system of equations that can be solved. In Section 8, we discuss in detail several aspects of our model which lead to the corresponding parameters used to predict the electrocapillarity curves in Figure 1.

\section{Thermodynamical consistent model for the equilibrium state}

In this section, we summarize the underlying thermodynamical consistent complete continuum model that spatially resolves boundary layers, without giving a detailed derivation of the model here. This model is the basis from which we can derive reduced models which provide the Lippmann equation. Our modelling is based on the framework of nonequilibrium thermodynamics $[9,14,34]$ and its extensions to surfaces and the connection to electrodynamics $[6,35]$. A quite general model containing all relevant ingredients for planar surface is provided in [16], the case of curved surfaces can be found in [28]. The notation is summarized in Table 1 .

\subsection{Setup}

We consider a surface $S$ dividing a domain $\Omega \subseteq \mathbb{R}^{3}$ into the subdomains $\Omega^{+}$and $\Omega^{-}$. The normal $v$ to the surface $S$ always points from $\Omega^{-}$to $\Omega^{+}$. For quantities defined in $\Omega^{+}$or $\Omega^{-}$, there will often be corresponding quantities on $S$. As a convention the same letters are used for these quantities but the surface variables are indicated by a subscript $s$.

\subsection{Jumps at surfaces}

We introduce the boundary values and the jump of a generic function $u(x)$ in $\Omega^{ \pm}$at the surface $S$ as

$$
\left.u\right|_{S} ^{ \pm}=\lim _{x \in \Omega^{ \pm} \rightarrow S} u(x) \quad \text { and } \quad \llbracket u \rrbracket=\left.u\right|_{S} ^{+}-\left.u\right|_{S} ^{-} .
$$

In the case the function $u$ is not defined in either $\Omega^{+}$or in $\Omega^{-}$, we set the corresponding value in (2.1) to zero.

\subsection{Constituents}

In each of the two domains $\Omega^{+}$and $\Omega^{-}$and on the surface $S$, we consider a mixture of several constituents. In $\Omega^{ \pm}$, we denote the constituents by $\mathrm{A}_{\alpha}$ where $\alpha$ is taken from some index set $\mathcal{M}^{+}$and $\mathcal{M}^{-}$, respectively. We assume that $\mathcal{M}^{+}$and $\mathcal{M}^{-}$are disjoint, i.e., $\mathcal{M}^{+} \cap \mathcal{M}^{-}=\emptyset$ and refer to their union as $\mathcal{M}^{ \pm}=\mathcal{M}^{+} \cup \mathcal{M}^{-}$. The index set for the constituents on $S$ is denoted by $\mathcal{M}_{S}$. For each constituent $\mathrm{A}_{\alpha} \in \mathcal{M}^{ \pm}$in one of the 
Table 1. Summary of notations

\begin{tabular}{|c|c|c|c|c|c|}
\hline $\begin{array}{r}k_{B} \\
\varepsilon_{0}\end{array}$ & $\begin{array}{l}{[\mathrm{J} / \mathrm{K}]} \\
{[\mathrm{C} /(\mathrm{V} \mathrm{m})]}\end{array}$ & $\begin{array}{l}\text { Boltzmann constant } \\
\text { Electric constant }\end{array}$ & $\begin{array}{r}e_{0} \\
\chi\end{array}$ & {$[\mathrm{C}]$} & $\begin{array}{l}\text { Elementary charge } \\
\text { Susceptibility }\end{array}$ \\
\hline$v$ & & Nnormal vector & $k_{M}$ & {$[1 / \mathrm{m}]$} & Mean curvature \\
\hline$s_{\alpha}^{i}$ & & $\begin{array}{l}\text { Stoichiometric coef. } \\
\text { bulk reactions }\end{array}$ & $S_{\alpha}^{i}$ & & $\begin{array}{l}\text { Stoichiometric coef. } \\
\text { surface reactions }\end{array}$ \\
\hline$z_{\alpha}$ & & Charge number & & & \\
\hline$m_{\alpha}$ & {$[\mathrm{kg}]$} & Mass & & & \\
\hline$T$ & {$[\mathrm{~K}]$} & Bulk temperature & $T$ & {$[\mathrm{~K}]$} & Surface temperature \\
\hline$n_{\alpha}$ & {$\left[\mathrm{m}^{-3}\right]$} & Bulk number density & $n_{\alpha}$ & {$\left[\mathrm{m}^{-2}\right]$} & Surface number density \\
\hline$E$ & {$[\mathrm{~V} / \mathrm{m}]$} & Electric field & & & \\
\hline$\varphi$ & {$[\mathrm{V}]$} & Electrostatic potential & $\varphi$ & {$[\mathrm{V}]$} & Electrostatic surface potential \\
\hline$n^{\mathrm{F}}$ & {$\left[\mathrm{C} / \mathrm{m}^{3}\right]$} & (Free) charge density & $n_{s}^{n^{\mathrm{F}}}$ & {$\left[\mathrm{C} / \mathrm{m}^{2}\right]$} & Surface (free) charge density \\
\hline$\rho \psi$ & {$\left[\mathrm{J} / \mathrm{m}^{3}\right]$} & Free energy density & $\rho \psi$ & {$\left[\mathrm{J} / \mathrm{m}^{2}\right]$} & Surface free energy density \\
\hline$\mu_{\alpha}$ & {$[\mathrm{J}]$} & Chemical potential & $\begin{array}{l}s s \\
\mu_{\alpha}\end{array}$ & {$[\mathrm{J}]$} & Surface chemical potential \\
\hline$p$ & {$\left[\mathrm{~N} / \mathrm{m}^{2}\right]$} & Material pressure & $\gamma$ & {$[\mathrm{N} / \mathrm{m}]$} & Surface tension \\
\hline$\rho \boldsymbol{b}$ & {$[\mathrm{C}]$} & Force density gravitation & $\rho_{s}^{s}$ & {$[\mathrm{C}]$} & Surface force density gravitation \\
\hline$\Sigma$ & {$\left[\mathrm{N} / \mathrm{m}^{2}\right]$} & Total stress tensor & & & \\
\hline$Q$ & {$\left[\mathrm{C} / \mathrm{m}^{2}\right]$} & Double layer charge density & $\widetilde{q}$ & {$\left[\mathrm{C} / \mathrm{m}^{2}\right]$} & Boundary layer charge density \\
\hline$\gamma$ & {$[\mathrm{N} / \mathrm{m}]$} & Interfacial tension & $\tilde{\gamma}$ & {$[\mathrm{N} / \mathrm{m}]$} & Boundary layer tension \\
\hline
\end{tabular}

subdomains $\Omega^{ \pm}$, we assume there is a corresponding constituent present on the surface $S$, but in addition there may be some constituents that are exclusively present on $S$ due to chemical reaction, i.e., $\mathcal{M}_{S} \supseteq \mathcal{M}^{ \pm}$. A constituent $\mathrm{A}_{\alpha}$ has the (atomic) mass $m_{\alpha}$ and may be carrier of the charge $z_{\alpha} e_{0}$, where $z_{\alpha}$ is the charge number and $e_{0}$ is the elementary charge.

\subsection{Chemical reactions}

Among the constituents, we may have chemical reactions, of which we only consider linearly independent ones. There are $M$ reactions in each bulk phase and in addition there may be $M_{S}$ surface reactions of the general form

$$
\begin{array}{ll}
\sum_{\alpha \in \mathcal{M}^{ \pm}} a_{\alpha}^{i} \mathrm{~A}_{\alpha} \rightleftharpoons \sum_{\alpha \in \mathcal{M}^{ \pm}} b_{\alpha}^{i} \mathrm{~A}_{\alpha} & \text { for } i \in\{1, \cdots, M\} \\
\sum_{\alpha \in \mathcal{M}_{S}} a_{\alpha}^{i} \mathrm{~A}_{\alpha} \rightleftharpoons \sum_{\alpha \in \mathcal{M}_{S}} b_{\alpha}^{i} \mathrm{~A}_{\alpha} & \text { for } i \in\left\{1, \cdots, M_{S}\right\} .
\end{array}
$$

The constants $a_{\alpha}^{i}, b_{\alpha}^{i}$ are positive integers and $s_{\alpha}^{i}=b_{\alpha}^{i}-a_{\alpha}^{i}$ denote the stoichiometric coefficients of the reactions. Since charge and mass have to be conserved by every single 
reaction in the bulk and on the surface, we have

$$
\begin{aligned}
& \sum_{\alpha \in \mathcal{M}^{ \pm}} z_{\alpha} s_{\alpha}^{i}=0 \quad \text { and } \quad \sum_{\alpha \in \mathcal{M}_{S}} z_{\alpha} s_{\alpha}^{i}=0 \text {, } \\
& \sum_{\alpha \in \mathcal{M}^{ \pm}} m_{\alpha} s_{\alpha}^{i}=0 \quad \text { and } \quad \sum_{\alpha \in \mathcal{M}_{s}} m_{\alpha} s_{\alpha}^{i}=0 .
\end{aligned}
$$

\subsection{Thermodynamic state}

In equilibrium, the thermodynamic state in each point $\boldsymbol{x} \in \Omega^{ \pm}$is described by the number densities $n_{\alpha}$ of the constituents, the temperature $T$ and the electric field $\boldsymbol{E}$. The thermodynamic state of the surface $S$ is characterized by the number densities $n_{\alpha}$ of the surface constituents and the interfacial temperature $T$.

In equilibrium, the temperature $T$ in both domains is constant and continuous at the surface $S$, i.e., $T=\left.T\right|_{S} ^{ \pm}$; hence, the temperature can be considered as a parameter here.

In equilibrium, the electric field can be expressed in terms of the electrostatic potential by $\boldsymbol{E}=-\nabla \varphi$. We assume that the electrostatic potential is continuous at the surface $S$ such that the Maxwell equation $\llbracket \nabla \varphi \times v \rrbracket=0$ is satisfied,

$$
\varphi_{S}=\left.\varphi\right|_{S} ^{-}=\left.\varphi\right|_{S} ^{+}
$$

The new quantity $\varphi$ is called the electrostatic surface potential.

\subsection{General constitutive assumptions}

We assume that the susceptibility $\chi$ is constant in each sub-domain, i.e., $\chi=\chi_{ \pm}$in $\Omega^{ \pm}$. To cover a wide range of materials we assume free energy densities in $\Omega^{ \pm}$and on $S$ of the form

$$
\rho \psi=\rho \hat{\psi}\left(T, n_{0}, \ldots, n_{N}\right)-\chi \frac{\varepsilon_{0}}{2}|\nabla \varphi|^{2}, \quad \underset{s s}{\rho \psi}=\underset{s s}{\rho \hat{\psi}}\left(\underset{s}{T}, n_{s}, \ldots, n_{s} n_{N_{S}}\right),
$$

where $\rho \hat{\psi}$ and $\rho_{s} \hat{\psi}$ are assumed to be convex $C^{2}$ functions. The chemical potentials are defined by

$$
\mu_{\alpha}=\frac{\partial \rho \hat{\psi}}{\partial n_{\alpha}}, \quad \quad \mu_{\alpha}=\frac{\partial \rho \hat{\psi} s}{\partial n_{\alpha}} .
$$

We introduce the material pressure and the surface tension as

$$
p=-\rho \hat{\psi}+\sum_{\alpha \in \mathcal{M}^{ \pm}} n_{\alpha} \mu_{\alpha}, \quad \gamma_{s}=\rho_{s} \hat{\psi}-\sum_{\alpha \in \mathcal{M}_{S}} n_{s} \mu_{s},
$$

and further on refer to these equations as Gibbs-Duhem relations of the bulk and the surface, respectively. 


\subsection{Model equations and boundary conditions}

In equilibrium, the mass balances, momentum balance and Maxwell's equations in $\Omega^{ \pm}$ reduce to $[16,31]$

$$
\begin{aligned}
& \nabla\left(\mu_{\alpha}+z_{\alpha} e_{0} \varphi\right)=\boldsymbol{b} \quad \text { for } \alpha \in \mathcal{M}^{ \pm}, \\
& -(1+\chi) \varepsilon_{0} \Delta \varphi=n^{\mathrm{F}},
\end{aligned}
$$

where $n^{\mathrm{F}}=e_{0} \sum_{\alpha \in \mathcal{M}^{ \pm}} z_{\alpha} n_{\alpha}$ denotes the free charge density in $\Omega^{ \pm}$. A direct calculation shows that the momentum balance results from the equation system above and the Gibbs-Duhem relation $(2.7)_{\text {left }}$

$$
-\operatorname{div}(\Sigma)=\rho \boldsymbol{b}
$$

where $\rho \boldsymbol{b}$ is the force densities due to gravitation and $\Sigma$ is the total stress tensor consisting of a material and an electromagnetic contribution called Maxwell stress,

$$
\Sigma=-p \mathbf{1}+(1+\chi) \varepsilon_{0}\left(\nabla \varphi \otimes \nabla \varphi-\frac{1}{2}|\nabla \varphi|^{2} \mathbf{1}\right) .
$$

Due to the chemical reactions in the bulk the chemical potentials of the involved species is restricted by the law of mass action

$$
\sum_{\alpha \in \mathcal{M}^{ \pm}} s_{\alpha}^{i} \mu_{\alpha}=0, \quad \text { for } \quad i \in\{1, \ldots, M\}
$$

The boundary conditions at $S$, which follow from surface balance equations, are $[28,35]$

$$
\begin{aligned}
& \left.\mu_{\alpha}\right|_{S} ^{ \pm}=\mu_{\alpha} \quad \text { for } \alpha \in \mathcal{M}^{ \pm}, \\
& -\llbracket \Sigma \cdot \boldsymbol{v} \rrbracket=2 k_{M} \underset{s}{\gamma \boldsymbol{v}}+{\underset{s}{\rho} \boldsymbol{b}}_{s}+\underset{s}{\nabla} \gamma_{s}, \\
& \llbracket \nabla \varphi \cdot v \rrbracket=n_{s}^{\mathrm{F}},
\end{aligned}
$$

where $\nabla_{s}$ denotes the tangential gradient on $S$ and $n_{s}^{\mathrm{F}}=e_{0} \sum_{\alpha \in \mathcal{M}_{S}} z_{\alpha} n_{\alpha}$ is the surface free charge density. The number densities of the surface constituents are restricted by the reaction constraints

$$
\sum_{\alpha \in \mathcal{M}_{S}} s_{S}^{i} \mu_{s}=0, \quad \text { for } \quad i \in\left\{1, \ldots, M_{S}\right\}
$$

\subsection{Conditions related to experimental setup}

For unique solvability of the equilibrium system, more conditions are needed in addition to (2.8)-(2.11). These conditions depend on the systems properties far away from the surface, and we do not want to detail them here. Instead we motivate conditions that are sufficient in the context of the Lippmann equation. In a typical experiment, the electrostatic potential is only measured at certain points far away from the surface, e.g., 


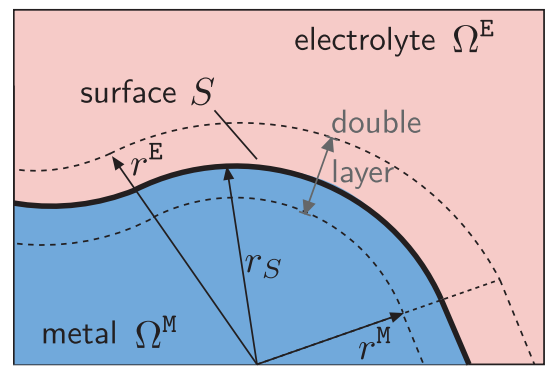

FIGURE 2. Sketch of double layer at a curved surface $S$ with local radial symmetry.

with reference electrodes. Moreover, if the domains are sufficiently large, the experiments do not show an influence of the electrostatic potential on the number densities far away from the surface. Thus, we assume in each domain $\Omega^{ \pm}$the existence of a point $x^{ \pm}$ sufficiently far away from the surface $S$, where the number densities $n_{\alpha}$ and the potential $\varphi$ can be prescribed independently, as long as this choice is consistent with (2.9).

\section{Motivation}

Within the above model, we cannot establish a relation like the Lippmann equation. There even is no well-defined potential difference in this model that could be used for this purpose. A sharp definition of the quantities in the Lippmann equation (1.1) is not that obvious as it might seem. While the Young-Laplace equation (1.2) might appear as an appropriate definition of the interfacial tension, in general such a relation does not exist in electro-thermodynamics. Only in the case of vanishing electric field, i.e., $\boldsymbol{E}=0$, and under some restriction on the surface and bulk stress tensor the surface momentum balance equations simplifies to (1.2). By the use of a simplified example, we want to motivate that also in the case of a non-zero electric field a variant of the Young-Laplace equation (1.2) can be derived.

We consider a liquid metal located in $\Omega^{\mathrm{M}}$ in contact with a liquid electrolyte in $\Omega^{\mathrm{E}}$ with some applied potential difference across the dividing interface $S$. In thermodynamic equilibrium, the electric field and the stress in the double layer is described by the coupled system of Poisson equation and the momentum balance

$$
\begin{aligned}
\operatorname{div}\left((1+\chi) \varepsilon_{0} \boldsymbol{E}\right) & =n^{\mathrm{F}} & \llbracket(1+\chi) \varepsilon_{0} \boldsymbol{E} \cdot \boldsymbol{v} \rrbracket & =n_{s}^{\mathrm{F}} \\
\operatorname{div}(\Sigma) & =0 & \llbracket \Sigma \cdot \boldsymbol{v} \rrbracket & =-2 k_{M} \gamma \boldsymbol{v}-\underset{s}{\gamma_{s}} .
\end{aligned}
$$

The total stress is given by

$$
\Sigma=-p \mathbf{1}+(1+\chi) \varepsilon_{0}\left(\boldsymbol{E} \otimes \boldsymbol{E}-\frac{1}{2}|\boldsymbol{E}|^{2} \mathbf{1}\right)
$$

Let us assume local spherical symmetry in the neighbourhood of some point on the surface as illustrated in Figure 2. Using spherical coordinates $(r, \theta, \psi)$ with basis vectors $\left(\boldsymbol{e}_{r}, \boldsymbol{e}_{\theta}, \boldsymbol{e}_{\psi}\right)$, the surface $S$ is characterized by $r=r_{S}$. Let $\Omega^{\mathrm{M}}$ be such that $r<r_{S}$ for points located in the metal. Due to the symmetry, we have $\boldsymbol{E}=E_{r} \boldsymbol{e}_{r}$, and the total stress tensor 
reduces to

$$
\Sigma=\Sigma_{r r} \boldsymbol{e}_{r} \otimes \boldsymbol{e}_{r}+\Sigma_{\theta \theta} \boldsymbol{e}_{\theta} \otimes \boldsymbol{e}_{\theta}+\Sigma_{\psi \psi} \boldsymbol{e}_{\psi} \otimes \boldsymbol{e}_{\psi}
$$

with

$$
\Sigma_{r r}=-p+\frac{1+\chi}{2} \varepsilon_{0} E_{r}^{2}, \quad \Sigma_{\theta \theta}=\Sigma_{\psi \psi}=-p-\frac{1+\chi}{2} \varepsilon_{0} E_{r}^{2} .
$$

In the considered neighbourhood, the Poisson-momentum equation system reduce to

$$
\begin{aligned}
\partial_{r}\left(r^{2}(1+\chi) \varepsilon_{0} E_{r}\right) & =r^{2} n^{\mathrm{F}}, & \llbracket(1+\chi) \varepsilon_{0} E_{r} \rrbracket & =n_{s}^{\mathrm{F}}, \\
\partial_{r} \Sigma_{r r} & =-\frac{2}{r}(1+\chi) \varepsilon_{0} E_{r}^{2}, & \llbracket \Sigma_{r r} \rrbracket & =-\frac{2}{r_{s}} \gamma_{s}
\end{aligned}
$$

The electric field typically vanishes a few nanometres away from the interface. Let $r^{\mathrm{M}}<r_{S}<r^{\mathrm{E}}$ such that $E_{r}=0$ for $r=r^{\mathrm{E} / \mathrm{M}}$ and assume that the mean curvature $k_{M}=-1 / r_{S}$ is small enough such that $\left|r k_{M}\right| \approx 1$ for $r \in\left(r^{\mathrm{M}}, r^{\mathrm{E}}\right)$. Then, we can approximate the integration of the bulk equations (3.6) $)_{1}$ and (3.7) $)_{1}$ with respect to $r$ by

$$
\begin{aligned}
-\left(1+\chi_{\mathrm{M} / \mathrm{E}}\right) \varepsilon_{0}\left(\left.E_{r}\right|_{r_{S}} ^{\Omega^{\mathrm{M} / \mathrm{E}}}-\left.E_{r}\right|_{r^{\mathrm{M} / \mathrm{E}}}\right) & =\operatorname{sgn}\left(r^{\mathrm{M} / \mathrm{E}}-r_{S}\right) q_{\mathrm{BL}}^{\mathrm{M} / \mathrm{E}}, \\
-\left(\left.\Sigma_{r r}\right|_{r_{S}^{\mathrm{M} / \mathrm{E}}}-\left.\Sigma_{r r}\right|_{r^{\mathrm{M} / \mathrm{E}}}\right) & =\operatorname{sgn}\left(r^{\mathrm{M} / \mathrm{E}}-r_{S}\right) 2 k_{M} \gamma_{\mathrm{BL}}^{\mathrm{M} / \mathrm{E}} .
\end{aligned}
$$

Here, we introduced new quantities in the metal and in the electrolyte phase that we refer to as boundary layer charge density and boundary layer tension, i.e.,

$$
q_{\mathrm{BL}}^{\mathrm{M} / \mathrm{E}}=\operatorname{sgn}\left(r^{\mathrm{M} / \mathrm{E}}-r_{S}\right) \int_{r_{S}}^{r^{\mathrm{M} / \mathrm{E}}} n^{\mathrm{F}} d r, \quad \gamma_{\mathrm{BL}}^{\mathrm{M} / \mathrm{E}}=\operatorname{sgn}\left(r^{\mathrm{M} / \mathrm{E}}-r_{S}\right) \int_{r_{S}}^{r^{\mathrm{M} / \mathrm{E}}}\left(1+\chi_{\mathrm{M} / \mathrm{E}}\right) \varepsilon_{0} E_{r}^{2} d r .
$$

The boundary conditions $(3.6)_{2}$ and $(3.7)_{2}$ can be used to connect (3.8) and (3.9) for $\Omega^{\mathrm{M}}$ and $\Omega^{\mathrm{E}}$. With $\left.\Sigma_{r r}\right|_{r^{\mathrm{M} / \mathrm{E}}}=-\left.p\right|_{r^{\mathrm{M} / \mathrm{E}}}$ due to $\left.E_{r}\right|_{r^{\mathrm{M} / \mathrm{E}}}=0$, we conclude

$$
\begin{gathered}
0=n_{s}^{\mathrm{F}}+q_{\mathrm{BL}}^{\mathrm{E}}+q_{\mathrm{BL}}^{\mathrm{M}}, \\
\left.p\right|_{r^{\mathrm{E}}}-\left.p\right|_{r^{\mathrm{M}}}=2 k_{M}\left(\gamma_{s}-\gamma_{\mathrm{BL}}^{\mathrm{M}}-\gamma_{\mathrm{BL}}^{\mathrm{E}}\right) .
\end{gathered}
$$

While the electroneutrality condition of the electrical double layer (3.11) could have been expected, the second result (3.12) is quite remarkable. It states that the pressure jump across the entire electrical double layer does not only depend on the thermodynamic surface tension $\gamma$, but also on the boundary layer tension generated by electric field in the space charge layers. Upon defining the interfacial tension of the electrical double layer as

$$
\gamma:=\gamma_{s}-\gamma_{\mathrm{BL}}^{\mathrm{M}}-\gamma_{\mathrm{BL}}^{\mathrm{E}}
$$

the equation (3.12) has the structure of the Young-Laplace equation (1.2). It seems reasonable to expect that the Lippmann equation relates this interfacial tension $\gamma$ to the voltage between $r=r^{\mathrm{M}}$ and $r=r^{\mathrm{E}}$ and the total charge stored in this double layer in 
between. Moreover, we see that charging the boundary layer always causes non-negative contributions $\gamma_{\mathrm{BL}}^{\mathrm{M} / \mathrm{E}}$ that lower the interfacial tension $\gamma$ away from the potential of zero charge. This directly explains the U- or parabola-shaped electrocapillary curves that are observed in experiments.

\section{Reduced models and Lippmann equation}

When two electrochemical systems are brought into contact it is well-known that narrow boundary layers are formed adjacent to the contact surface. The width of the layers is in the order of the Debye-length, which for liquid electrolytes is usually in the range of nanometres. If the macroscopic size $L^{r e f}$ of the system is in the range of centimetrs, one can introduce a small dimensionless number $\lambda$ to represent the Debye length as $\lambda L^{r e f}$. Based on asymptotic analysis of complete thermodynamic model above, we derive in Section 5 a set of reduced models to describe the limit of thin double layers. These reduced models are as follows: (i) the leading order bulk model, (ii) the first higher order bulk model, and (iii) the leading order surface and boundary layer models. The reduced models then allow to derive a Lippmann equation in a very general form. The derivation is carried out in Section 6.

In this section, we summarize the reduced models for the thin interface limit and then state the Lippmann equation. We prefer to apply a different notation for the jumps at the interface here to highlight the interpretation as jumps over the complete double layer. We denote the leading order parameterization of the surface $S$ by $I$ and define for a generic function $u^{(0)}$ on the regions $\Omega^{ \pm}$

$$
\left.u^{(0)}\right|_{I} ^{ \pm}=\lim _{x \rightarrow I^{ \pm}} u^{(0)} \quad \text { and } \quad \llbracket u^{(0)} \mathbb{\mathbb { }}=\left.u^{(0)}\right|_{I} ^{+}-\left.u^{(0)}\right|_{I} ^{-} .
$$

The general constitutive assumptions are analogous to (2.5)-(2.7) above with the only difference that in the bulk phases the leading order free energy density simplifies to

$$
\rho \psi^{(0)}=\rho \hat{\psi}^{(0)}\left(T, n_{0}^{(0)}, \ldots, n_{N}^{(0)}\right) .
$$

\subsection{Constant leading order bulk quantities}

In each of the subdomains $\Omega^{ \pm}$, we have local electroneutrality

$$
n^{\mathrm{F},(0)}=0 \text {. }
$$

We have constant number densities $n_{\alpha}^{(0), \pm}$ and hence constant chemical potentials $\mu_{\alpha}^{(0), \pm}$ in $\Omega^{ \pm}$. Moreover, the electrostatic potential $\varphi^{(0), \pm}$ is constant in $\Omega^{ \pm}$and hence, there is a well-defined electric potential difference over the interface $I$. Moreover, the leading order of the surface momentum balance equations

$$
\mathbb{m} p^{(0)} \mathbb{\prod}=0 \quad \text { at } I,
$$

implies that in leading order the pressure $p^{(0)}$ is constant in $\Omega$. Thus, gravitation and surface tension have to be considered as higher order effects. There is no influence of the 
domain geometry on the leading order bulk system at all. Due the bulk reactions, the number densities are restricted by the mass action law, which reads in the leading order

$$
\sum_{\alpha \in \mathcal{M}^{ \pm}} s_{\alpha}^{i} \mu_{\alpha}^{(0)}=0, \quad \text { for } \quad i \in\{1, \ldots, M\} .
$$

The constant electric potential and number densities justify the experimental procedure to measure the bulk quantities somewhere in the domains $\Omega^{ \pm}$, as long as the measuring points are sufficiently far away from the surface $S$. In addition, experimental experience suggests that the bulk number densities $n_{\alpha}^{(0), \pm}$ are independent of the applied potential $\varphi^{(0, \pm)}$, as we assumed in Section 2.

\subsection{Surface and boundary layer equations of the leading order}

Given the input data $\varphi^{(0), \pm}$ and $n_{\alpha}^{(0), \pm}$ from the leading order problem, we can determine the surface number densities $n_{\alpha}^{(0)}$ and the surface electrostatic potential $\varphi_{s}^{(0)}$ as well as $\widetilde{\varphi}^{(0)}$, $\widetilde{n}_{\alpha}^{(0)}$ in the boundary layers.

\subsubsection{Surface}

The number densities ${ }_{s}^{(0)}$ and $\varphi_{s}^{(0)}$ are determined by

$$
\begin{aligned}
& \mu_{\alpha}^{(0), \pm}+z_{\alpha} e_{0} \varphi^{(0), \pm}=\underset{s}{\mu_{\alpha}^{(0)}}+z_{\alpha} e_{0} \varphi_{s}^{(0)} \quad \text { at } I \quad \text { for } \alpha \in \mathcal{M}^{ \pm} \text {, } \\
& 0=n_{s}^{\mathrm{F},(0)}+\widetilde{q}^{+}+\widetilde{q}^{-} \quad \text { at } I,
\end{aligned}
$$

where the boundary layer charge densities are defined as functions of boundary layer quantities below, viz.,

$$
\widetilde{q}^{ \pm}= \pm \int_{0}^{ \pm \infty} \widetilde{n}^{\mathrm{F},(0)} d x
$$

The constituents on the surface are restricted by the law of mass action, viz.,

$$
\sum_{\alpha \in \mathcal{M}_{S}} s_{S^{\prime}}^{i} \mu_{\alpha}^{(0)}=0, \quad \text { for } \quad i \in\left\{1, \ldots, M_{S}\right\} .
$$

\subsubsection{Boundary layer}

In the boundary layer, we only have to solve differential equations in one space dimension which we denote by $z$, i.e.,

$$
\begin{aligned}
\partial_{z}\left(\widetilde{\mu}_{\alpha}^{(0)}+z_{\alpha} e_{0} \widetilde{\varphi}^{(0)}\right) & =0, \quad \text { for } \alpha \in \mathcal{M}^{ \pm}, \\
-(1+\chi) \varepsilon_{0} \partial_{z z} \widetilde{\varphi}^{(0)} & =\widetilde{n}^{\mathrm{F},(0)},
\end{aligned}
$$


with boundary conditions

$$
\begin{array}{ll}
\lim _{z \rightarrow \pm \infty} \widetilde{n}_{\alpha}^{(0)}=n_{\alpha}^{(0), \pm}, & \text { for } \alpha \in \mathcal{M}^{ \pm}, \\
\lim _{z \rightarrow \pm \infty} \widetilde{\varphi}^{(0)}=\varphi^{(0), \pm}, & \text { and }\left.\quad \widetilde{\varphi}^{(0)}\right|_{z=0} ^{ \pm}=\varphi_{s}^{(0)}
\end{array}
$$

The boundary layer equations and the constant bulk quantities of the leading order imply that $\varphi_{s}^{(0)}$ is independent of the space coordinates and thus there is a well-defined potential difference between the surface and each of the bulk domains $\Omega^{ \pm}$in leading order.

In the boundary layer, the momentum balance has the representation

$$
\partial_{z} \widetilde{p}^{(0)}+\widetilde{n}^{\mathrm{F},(0)} \partial_{z} \widetilde{\varphi}^{(0)}=0
$$

We define the quantities

$$
\tilde{\gamma}^{ \pm}= \pm \int_{0}^{ \pm \infty}(1+\chi) \varepsilon_{0}\left|\partial_{z} \widetilde{\varphi}^{(0)}\right|^{2} d x
$$

as boundary layer tensions. The meaning of this definition becomes accessible in the following section.

\subsection{Higher order bulk and surface relations}

The variables in first order are the electrostatic potential $\varphi^{(1)}$ and the number densities $n_{\alpha}^{(1)}$. They are related to the chemical potentials as $\mu_{\alpha}^{(1)}=\sum_{\beta}\left(\frac{\partial \mu_{\alpha}}{\partial n_{\beta}}\right)^{(0)} n_{\beta}^{(1)}$. The governing equations in $\Omega^{ \pm}$are

$$
\begin{aligned}
\nabla\left(\mu_{\alpha}^{(1)}+z_{\alpha} e_{0} \varphi^{(1)}\right) & =\boldsymbol{b} \quad \text { for } \alpha \in \mathcal{M}^{ \pm} \\
0 & =n^{\mathrm{F},(1)}
\end{aligned}
$$

From these equations and the Gibbs-Duhem equation (2.7) left in the first order, the momentum balance follows as

$$
\nabla p^{(1)}=\rho^{(0)} \boldsymbol{b}
$$

Thus, due to gravitation the pressure as well as the electrochemical potentials are not constant in the first higher order.

The jump condition for the pressure in the first order at the thin double layer interface $I$ is

$$
\mathbb{m} p^{(1)} \mathbb{\prod}=2 k_{M}^{(0)}\left(\gamma_{s}^{(0)}-\widetilde{\gamma}^{+}-\widetilde{\gamma}^{-}\right)
$$

where $\gamma^{(0)}$ is the surface tension given by the Gibbs-Duhem equation $(2.7)_{\text {right }}$ in the leading order and the boundary layer tensions $\widetilde{\gamma}^{ \pm}$are defined in (4.14). 


\subsection{Lippmann equation}

Within the reduced models above, it is possible to define a potential difference $U$, a double layer charge density $Q$ and the interfacial tension $\gamma$ such that

$$
\frac{d}{d U} \gamma=-Q
$$

In particular this Lippmann equation (4.18) holds independent of any specific material model and for a wide range of admissible surface reactions, provided that the electric potential in the boundary layers varies in a monotonic way in normal direction.

The definition of the double layer charge density requires some restrictions on the set $\mathcal{M}_{S} \backslash \mathcal{M}^{ \pm}$of exclusive surface species and the admissible surface reactions. We neglect exclusive surface species that do not participate in any surface reaction, and we exclude surface reactions where no bulk species are involved. Therefore, we assume that there are $M_{S}$ exclusive surface species $\mathrm{A}_{\alpha} \in \mathcal{M}_{S} \backslash \mathcal{M}^{ \pm}$and each of them can be related to the bulk species by at least one of the (linear independent) surface reactions. ${ }^{1}$ We thus consider $M_{S}$ surface reactions of the form

$$
\mathrm{A}_{\beta} \rightleftharpoons \sum_{\alpha \in \mathcal{M}^{ \pm}} v_{\alpha \beta} \mathrm{A}_{\alpha} \quad \text { for } \beta \in \mathcal{M}_{S} \backslash \mathcal{M}^{ \pm}
$$

Using the charge neutrality $(2.3 a)$ for the surface reactions, the reaction equilibrium condition (4.8) can be written as

$$
\underset{s}{\mu_{\beta}^{(0)}}+\underset{\beta}{z_{\beta} e_{0} \varphi^{(0)}}=\sum_{\alpha \in \mathcal{M}^{ \pm}} v_{\alpha \beta}\left(\underset{s}{\mu_{\alpha}^{(0)}}+\underset{\alpha}{\left.z_{\alpha} e_{0} \varphi^{(0)}\right)} \quad \text { for } \beta \in \mathcal{M}_{S} \backslash \mathcal{M}^{ \pm}\right.
$$

Then, we define the double layer charge density as

$$
Q:=-\widetilde{q}^{-}-\sum_{\alpha \in \mathcal{M}^{-}} z_{\alpha} e_{0} n_{\alpha}^{(0)}-\sum_{\left(\alpha \in \mathcal{M}^{-}\right)} \sum_{\left(\beta \in \mathcal{M}_{S} \backslash \mathcal{M}^{ \pm}\right)} v_{\alpha \beta} z_{\beta} e_{0} n_{s}^{(0)},
$$

where in addition to the expected charge contribution from surface and layer, there is one more term that results from the surface reactions. An experimental verification of the Lippmann equation is possible, if the surface charge $Q$ can be related to the electric current into an electrode. In this case, it is obvious that also reactants of the surface reactions contribute to the current into the electrode and thus to the surface charge. For the liquid metal-electrolyte interface the current-charge relation (7.17) is derived in Appendix B.

Because the electric potential in $\Omega^{ \pm}$in leading order is independent of the space variable, there is a well-defined potential difference

$$
U:=\varphi^{(0),+}-\varphi^{(0),-} .
$$

1 By this assumption, we allow for surface reactions like solvation shell stripping and autoprotolysis of the solvent. Also, electron transfer reactions are admissible, as far as the reaction products remain on the surface. Not allowed are charge transfer reactions between the bulk phases. 
Table 2. Substitution in the bulk regions $\Omega^{ \pm}$

$$
\begin{aligned}
& \boldsymbol{x} \rightarrow L^{r e f} \boldsymbol{x} \quad m_{\alpha} \rightarrow m^{r e f} m_{\alpha} \quad n_{\alpha} \rightarrow n^{r e f} n_{\alpha} \\
& \varphi \rightarrow \frac{k_{B} T}{e_{0}} \varphi \quad \boldsymbol{b} \rightarrow \frac{k_{B} T}{m^{\text {ref }} L^{r e f}} \lambda \boldsymbol{b} \\
& \rho \psi \rightarrow n^{r e f} k_{B} T \rho \psi \quad \quad \mu_{\alpha} \rightarrow k_{B} T \mu_{\alpha} \quad \Sigma \rightarrow n^{r e f} k_{B} T \Sigma \quad p \rightarrow n^{r e f} k_{B} T p
\end{aligned}
$$

Table 3. Substitution on the surface $S$

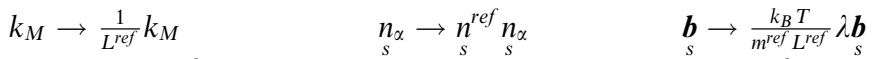

$$
\begin{aligned}
& \underset{s s}{\rho \psi} \rightarrow k_{B} T n_{s}^{r e f} \rho_{s s} \psi \quad \mu_{s} \rightarrow k_{B} T \mu_{s} \quad \gamma_{s} \rightarrow n_{s}^{r e f} k_{B} T \gamma_{s}^{s}
\end{aligned}
$$

According to (4.4), the pressure in the leading order is continuous across the double layer. Thus, the first relevant contributions have to be of higher order. In the first order, the jump of the pressure is given by the Young-Laplace equation (4.17), where the interfacial tension $\gamma$ of the reduced model is composed of the thermodynamic surface tension $\gamma_{s}^{(0)}$ and two electromagnetic contributions $\widetilde{\gamma}^{ \pm}$, viz.,

$$
\gamma:=\gamma_{s}^{(0)}-\widetilde{\gamma}^{+}-\widetilde{\gamma}^{-}
$$

\section{Mathematical derivation of the reduced models}

We use the method of formal asymptotic analysis to derive for a curved interface from the complete model of Section 2 the reduced models of Section 4. Formal asymptotic analysis has been applied to electrochemical systems, e.g., by $[4,5,11,17,26,36,40,42]$, and we refer to [16] for a detailed description of our approach in the planar case.

\subsection{Summary of model equations in dimensionless form}

We introduce scaling constants $L^{r e f}, n^{r e f}$, and $m^{\text {ref }}$ that are related to characteristic length, particle density and molecular weight in the system and introduce a characteristic surface particle density $n_{S}^{\text {ref }}$ on the surface $S$. For the non-dimensionalization of the model equations, we substitute the variables according to Tables 2 and 3.

This scaling generates the dimensionless numbers

$$
\lambda=\sqrt{\frac{\varepsilon_{0} k_{B} T}{e_{0}^{2} n^{r e f}\left(L^{r e f}\right)^{2}}}, \quad \lambda \delta=\frac{n^{r e f}}{n^{r e f} L^{r e f}} .
$$

Then, the dimensionless version of the model equations reads

$$
\begin{aligned}
\nabla\left(\mu_{\alpha}+z_{\alpha} \varphi\right) & =\lambda \boldsymbol{b} \quad \text { for } \alpha \in \mathcal{M}^{ \pm}, \\
-\lambda^{2}(1+\chi) \Delta \varphi & =n^{\mathrm{F}} .
\end{aligned}
$$


The dimensionless jump conditions on the surface $S$ are represented by

$$
\begin{aligned}
& \left.\left(\mu_{\alpha}+z_{\alpha} \varphi\right)\right|_{S} ^{ \pm}=\underset{s}{\left(\mu_{\alpha}+z_{\alpha} \varphi\right)} \quad \text { for } \alpha \in \mathcal{M}^{ \pm},
\end{aligned}
$$

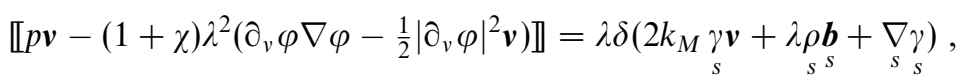

$$
\begin{aligned}
& -\llbracket \lambda(1+\chi) \partial_{v} \varphi \rrbracket=\delta n_{s}^{\mathrm{F}} .
\end{aligned}
$$

Pressure and surface tension are given by

$$
p=-\rho \psi+\sum_{\alpha \in \mathcal{M}^{ \pm}} n_{\alpha} \mu_{\alpha}, \quad \gamma_{s}=\rho_{s} \psi-\sum_{\alpha \in \mathcal{M}_{S}} n_{s} \mu_{s} \mu_{\alpha} .
$$

and the momentum balance can be recovered from (5.2a) and (5.4), i.e.,

$$
\nabla p+n^{\mathrm{F}} \nabla \varphi=\lambda \rho \boldsymbol{b}
$$

\subsubsection{Discussion of the dimensionless numbers}

We choose as characteristic number densities in the bulk and on the surface

$$
n^{r e f}=6.022 \cdot 10^{25} \mathrm{~m}^{-3}, \quad n_{s}^{r e f}=7.3 \cdot 10^{18} \mathrm{~m}^{-2},
$$

which corresponds to a 0.1 molar aqueous solution and a typical spacing of the crystal lattice of a metal. As room temperature and a characteristic mass, we choose

$$
T=298.15 \mathrm{~K}, \quad m^{r e f}=1.66 \cdot 10^{-27} \mathrm{~kg} .
$$

Given a characteristic length of the macroscopic system of

$$
L^{r e f}=10^{-2} \mathrm{~m}
$$

and with the standard gravity of Earth $g=9.81 \mathrm{~ms}^{-2}$, we get the dimensionless numbers

$$
\lambda \approx 1.54 \cdot 10^{-8}, \quad \lambda \delta \approx 1.21 \cdot 10^{-5}, \quad \lambda|\boldsymbol{b}| \approx 2.38 \cdot 10^{-8} .
$$

Then, we can use $\lambda$ as a single smallness parameter in the system, whereas $\delta$ and $\boldsymbol{b}$ are considered as quantities of moderate size.

\subsection{Formal asymptotic expansions}

Since the solution of the above complete model (5.2)-(5.4) depends on the parameter $\lambda$ relating the different length scales in the system, we add in the following an upper index $\lambda$ to all these functions. Let $u^{\lambda}$ be a generic function from our list of state variables in $\Omega^{ \pm}$. We assume $\lambda \ll 1$ and approximate $u^{\lambda}$ in the bulk by anter expansion with respect to the small parameter

$$
u^{\lambda}=u^{(0)}+\lambda u^{(1)}+\lambda^{2} u^{(2)}+\ldots
$$


where the newly introduced functions $u^{(0)}, u^{(1)}, u^{(2)}, \ldots$ still need to be determined. For a function $F$ of $u^{\lambda}$, the expansion is given by a Taylor series

$$
F\left(u^{\lambda}\right)=F\left(u^{(0)}\right)+\lambda F^{\prime}\left(u^{(0)}\right) u^{(1)}+\mathcal{O}\left(\lambda^{2}\right) .
$$

We use the abbreviations $F^{(0)}=F\left(u^{(0)}\right)$ and $F^{(1)}=F^{\prime}\left(u^{(0)}\right) u^{(1)}$ for the leading and higher order terms. In analogous way, we introduce expansions of the state variables on surface and for functions thereof.

If $\lambda \ll 1$, the boundary layer constitutes only a small portion of the domains $\Omega^{ \pm}$and the outer expansion does not necessarily have to be accurate inside the layers. Therefore, we introduce an additional inner expansion inside the layer, which is based on space coordinates that are re-scaled by $\lambda$ in the normal direction. To distinguish between the two expansions in the bulk and in the boundary layer, we denote the inner expansion by $\widetilde{u}^{\lambda}$ and write

$$
\widetilde{u}^{\lambda}=\widetilde{u}^{(0)}+\lambda \widetilde{u}^{(1)}+\lambda^{2} \widetilde{u}^{(2)}+\ldots
$$

The two approximations have to be related by so called matching conditions which are detailed in Section 5.5 below. While the variables in the inner expansion have to satisfy the boundary conditions at $S$, for the outer expansion the role of the boundary conditions is taken by the matching conditions.

\subsection{Bulk equations}

\subsubsection{Leading order}

From (5.2), we can directly read off the leading order bulk equation in $\Omega^{ \pm}$

$$
\begin{aligned}
\nabla\left(\mu_{\alpha}^{(0)}+z_{\alpha} \varphi^{(0)}\right) & =0 \quad \text { for } \alpha \in \mathcal{M}^{ \pm}, \\
0 & =n^{\mathrm{F},(0)}
\end{aligned}
$$

Without loss of generality we assume $z_{N} \neq 0$ in $\Omega^{-}$. Then, we deduce from $(5.13 a)$

$$
\nabla\left(\mu_{\alpha}^{(0)}-\frac{z_{\alpha}}{z_{N}} \mu_{N}^{(0)}\right)=0 \quad \text { for } \alpha \in \mathcal{M}^{ \pm} \backslash\{N\},
$$

Multiplication with $\nabla n_{\alpha}^{(0)}$ and summation yields

$$
\sum_{\alpha=0}^{N-1} \sum_{\beta=0}^{N-1}\left(\frac{\partial \mu_{\alpha}^{(0)}}{\partial n_{\beta}^{(0)}}-\frac{z_{\beta}}{z_{N}} \frac{\partial \mu_{\alpha}^{(0)}}{\partial n_{N}^{(0)}}-\frac{z_{\alpha}}{z_{N}} \frac{\partial \mu_{N}^{(0)}}{\partial n_{\beta}^{(0)}}+\frac{z_{\alpha}}{z_{N}} \frac{z_{\beta}}{z_{N}} \frac{\partial \mu_{N}^{(0)}}{\partial n_{N}^{(0)}}\right) \nabla n_{\beta}^{(0)} \nabla n_{\alpha}^{(0)}=0,
$$

where $n_{N}^{(0)}$ has been eliminated by $(5.13 b)$. Since by assumption $\rho \hat{\psi}$ is convex, the matrix $\left(\frac{\partial \mu_{\alpha}^{(0)}}{\partial n_{\beta}^{(0)}}\right)_{\alpha, \beta}$ is symmetric and positive definite. Multiplying this matrix from left and right by the vector $\left(\nabla n_{0}^{(0)}, \cdots, \nabla n_{N-1}^{(0)},-\sum_{\beta=0}^{N-1} \frac{z_{\beta}}{z_{N}} \nabla n_{\beta}^{(0)}\right)$, yields (5.15). Therefore (5.15) and (5.13b) imply $\nabla n_{\alpha}^{(0)}=0$ for $\alpha \in \mathcal{M}^{ \pm}$. And, from (5.13a), we conclude $\nabla \varphi^{(0)}=0$. As a consequence, we see that $\varphi^{(0)}$ and all $n_{\alpha}^{(0)}$ are constant in $\Omega^{-}$. By analogy the same holds true also in $\Omega^{+}$. 
In the leading order of the momentum balance (5.5), the force densities due to gravitation does not contribute, and since $n^{F,(0)}=0$, the momentum balance simplifies to

$$
\nabla p^{(0)}=0
$$

Thus, also the pressure is constant in each of the sub-domains $\Omega^{ \pm}$.

\subsubsection{Higher order}

The bulk equations in the order $\mathcal{O}(\lambda)$ are

$$
\begin{aligned}
\nabla\left(\mu_{\alpha}^{(1)}+z_{\alpha} \varphi^{(1)}\right) & =\boldsymbol{b}, \\
0 & =n^{\mathrm{F},(1)} .
\end{aligned}
$$

and the momentum balance in higher order can be recovered as

$$
\nabla p^{(1)}=\rho^{(0)} \boldsymbol{b} .
$$

\subsection{Expansion of surface and boundary layers}

Locally, points on the surface $S$ can be represented as $\boldsymbol{r}\left(s_{1}, s_{2}\right)$. The partial derivatives $\partial_{1} \boldsymbol{r}$ and $\partial_{2} \boldsymbol{r}$ define the tangential vectors $\tau_{1}$ and $\tau_{2}$, respectively, which we assume to be orthogonal. In a neighbourhood $\mathcal{U}$ of a smooth surface $S$, the distance function is well defined. Each point $\boldsymbol{x} \in \mathcal{U}$ has a representation $\boldsymbol{x}=\boldsymbol{r}+z \boldsymbol{v}$, where $z$ is the distance to $S$. For a generic variable $u$ defined on $\mathcal{U}$, we introduce re-scaled inner variable $\widetilde{u}$ by defining

$$
\begin{aligned}
\widetilde{u}\left(s_{1}, s_{2}, z\right) & =u\left(\boldsymbol{r}\left(s_{1}, s_{2}\right)+\lambda z \boldsymbol{v}\right) . \\
\widetilde{u} & =\widetilde{u}^{(0)}+\lambda \widetilde{u}^{(1)}+\mathcal{O}\left(\lambda^{2}\right) .
\end{aligned}
$$

Moreover, we assume that the parameterization and the normal can be expanded as

$$
\begin{aligned}
& \boldsymbol{r}\left(s_{1}, s_{2}\right)=\boldsymbol{r}^{(0)}\left(s_{1}, s_{2}\right)+\lambda \boldsymbol{r}^{(1)}\left(s_{1}, s_{2}\right)+\mathcal{O}\left(\lambda^{2}\right), \\
& \boldsymbol{v}\left(s_{1}, s_{2}\right)=\boldsymbol{v}^{(0)}\left(s_{1}, s_{2}\right)+\lambda \boldsymbol{v}^{(1)}\left(s_{1}, s_{2}\right)+\mathcal{O}\left(\lambda^{2}\right),
\end{aligned}
$$

\subsubsection{Transformation of derivatives}

The re-scaling in normal direction leads to the following relations for the derivatives, cf. [15]:

$$
\begin{aligned}
\nabla u & =\lambda^{-1} \partial_{z} \widetilde{u} \boldsymbol{v}+\left|\tau_{\mathbf{1}}\right|^{-2} \partial_{1} \widetilde{u} \tau_{1}+\left|\tau_{\mathbf{2}}\right|^{-2} \partial_{2} \widetilde{u} \tau_{2}+\mathcal{O}(\lambda), \\
\operatorname{div}(\boldsymbol{u}) & =\lambda^{-1} \partial_{z} \widetilde{\boldsymbol{u}} \cdot \boldsymbol{v}+\operatorname{div}_{\tau}(\widetilde{\boldsymbol{u}})+\mathcal{O}(\lambda), \\
-\Delta u & =-\lambda^{-2} \partial_{z z} \widetilde{u}+\lambda^{-1} 2 k_{M} \partial_{z} \widetilde{u}+\mathcal{O}(1),
\end{aligned}
$$

where $\operatorname{div}_{\tau}$ denotes the surface divergence. If $S$ does not depend on $\lambda$, the $\mathcal{O}(\lambda)$ terms in $(5.22 a)$ and $(5.22 b)$ vanish. 


\subsubsection{Equations in inner variables}

The model equations in inner variables read

$$
\begin{aligned}
\left(\partial_{z} \widetilde{\mu}_{\alpha}+z_{\alpha} \partial_{z} \widetilde{\varphi}\right)+\mathcal{O}\left(\lambda^{2}\right) & =0, \\
\left(\partial_{1,2} \widetilde{\mu}_{\alpha}+z_{\alpha} \partial_{1,2} \widetilde{\varphi}\right)+\mathcal{O}(\lambda) & =0, \\
-(1+\chi)\left(\partial_{z z} \widetilde{\varphi}-\lambda 2 k_{M} \partial_{z} \widetilde{\varphi}\right)+\mathcal{O}\left(\lambda^{2}\right) & =\widetilde{n}^{\mathrm{F}} .
\end{aligned}
$$

The dimensionless jump conditions on the surface $S$ are represented by

$$
\begin{aligned}
& \left.\left(\widetilde{\mu}_{\alpha}+z_{\alpha} \widetilde{\varphi}\right)\right|_{S} ^{ \pm}=\underset{s}{\left(\mu_{\alpha}+z_{\alpha} \varphi\right),} \\
& \llbracket \widetilde{p}-\frac{1+\chi}{2}\left|\partial_{z} \widetilde{\varphi}\right|^{2} \rrbracket=\lambda \delta 2 k_{M} \gamma_{s}+\mathcal{O}\left(\lambda^{2}\right), \\
& -\llbracket(1+\chi) \partial_{z} \widetilde{\varphi} \partial_{1,2} \widetilde{\varphi} \rrbracket=\delta \partial_{1,2} \gamma+\mathcal{O}(\lambda), \\
& -\llbracket \lambda(1+\chi) \partial_{z} \widetilde{\varphi} \rrbracket=\delta \lambda n_{s}^{\mathrm{F}}+\mathcal{O}\left(\lambda^{2}\right) .
\end{aligned}
$$

Also in the layers, we can recover the momentum balance from (5.23) and the GibbsDuhem relation

$$
\begin{aligned}
\left(\partial_{z} \widetilde{p}+\widetilde{n}^{\mathrm{F}} \partial_{z} \widetilde{\varphi}\right)+\mathcal{O}\left(\lambda^{2}\right) & =0, \\
\left(\partial_{1,2} \widetilde{p}+\widetilde{n}^{\mathrm{F}} \partial_{1,2} \widetilde{\varphi}\right)+\mathcal{O}(\lambda) & =0,
\end{aligned}
$$

\subsubsection{Leading order system}

After solving the inner and the outer problem, it turns out that the inner tangential equations (5.23b), (5.26) and the surface equations (5.24c) do not contribute any additional independent information. Thus, they are omitted here. The remaining inner equations in leading order read

$$
\begin{aligned}
\partial_{z}\left(\widetilde{\mu}_{\alpha}^{(0)}+z_{\alpha} \widetilde{\varphi}^{(0)}\right) & =0, \\
\partial_{z} \widetilde{p}^{(0)}+\widetilde{n}^{\mathrm{F},(0)} \partial_{z} \widetilde{\varphi}^{(0)} & =0, \\
-(1+\chi) \partial_{z z} \widetilde{\varphi}^{(0)} & =\widetilde{n}^{\mathrm{F},(0)} .
\end{aligned}
$$

In particular, the inner electrochemical potentials are constant in leading order. The jump conditions in leading order are

$$
\begin{aligned}
& \left.\left(\widetilde{\mu}_{\alpha}^{(0)}+z_{\alpha} \widetilde{\varphi}^{(0)}\right)\right|_{z=0} ^{ \pm}=\mu_{\alpha}^{(0)}+z_{\alpha} \varphi_{s}^{(0)}, \\
& \llbracket \widetilde{p}^{(0)}-\frac{1+\chi}{2}\left|\partial_{z} \widetilde{\varphi}^{(0)}\right|^{2} \rrbracket=0, \\
& -\llbracket(1+\chi) \partial_{z} \widetilde{\varphi}^{(0)} \rrbracket=\delta n_{s}^{\mathrm{F},(0)} .
\end{aligned}
$$




\subsubsection{Higher order}

As in the leading order, the inner tangential equations and the surface equations can be omitted. The remaining first order of the equation system (5.23) is

$$
\begin{aligned}
\partial_{z}\left(\widetilde{\mu}_{\alpha}^{(1)}+z_{\alpha} \widetilde{\varphi}^{(1)}\right) & =0, \\
\partial_{z} \widetilde{p}^{(1)}+\widetilde{n}^{\mathrm{F},(0)} \partial_{z} \widetilde{\varphi}^{(1)}+\widetilde{n}^{\mathrm{F},(1)} \partial_{z} \widetilde{\varphi}^{(0)} & =0, \\
-(1+\chi)\left(\partial_{z z} \widetilde{\varphi}^{(1)}-2 k_{M}^{(0)} \partial_{z} \widetilde{\varphi}^{(0)}\right) & =\widetilde{n}^{\mathrm{F},(1)} .
\end{aligned}
$$

We see that the electrochemical potentials in the layers are also constant in the first order. The higher order jump conditions for the chemical potentials and the pressure are

$$
\begin{array}{r}
\left.\left(\widetilde{\mu}_{\alpha}^{(1)}+z_{\alpha} \widetilde{\varphi}^{(1)}\right)\right|_{z=0} ^{ \pm}=\left(\underset{s}{\left.\mu_{\alpha}^{(1)}+z_{\alpha} \varphi_{s}^{(1)}\right)}\right. \\
\llbracket \widetilde{p}^{(1)}-(1+\chi) \partial_{z} \widetilde{\varphi}^{(0)} \partial_{z} \widetilde{\varphi}^{(1)} \rrbracket=\delta 2 k_{M}^{(0)} \gamma_{s}^{(0)} .
\end{array}
$$

\subsection{Matching of inner and outer expansions}

Inner and outer expansions are related by so called matching conditions. In $[10,39]$, the matching conditions are formally achieved by inserting the corresponding expansions into the left and right hand sides of (5.21) and subsequent comparison of powers of $\lambda$. The result is, cf. [15]:

$$
\begin{aligned}
\widetilde{u}^{(0)}(z)-u^{(0), \pm}\left(\boldsymbol{r}^{(0)}\right) & =o(1 /|z|), \\
\partial_{z} \widetilde{u}^{(0)}(z) & =o(1 /|z|),
\end{aligned}
$$

and for the terms in higher order we get

$$
\begin{aligned}
\widetilde{u}^{(1)}(z)-z \partial_{v} u^{(0), \pm}\left(\boldsymbol{r}^{(0)}\right)-u^{(1), \pm}\left(\boldsymbol{r}^{(0)}\right) & =o(1 /|z|), \\
\partial_{z} \widetilde{u}^{(1)}(z)-\partial_{v} u^{(0), \pm}\left(\boldsymbol{r}^{(0)}\right) & =o(1 /|z|),
\end{aligned}
$$

Whenever a variable is constant inside the layer, we get by the matching conditions a relation of the boundary values from the outer expansion to the boundary values of the inner variables at $S$.

\subsubsection{Leading order}

Since the inner electrochemical potentials are constant according to $(5.27 a)$, the matching conditions can be used to relate the electrochemical potentials of the outer expansion to the boundary values of $(5.28 a)$, viz.,

$$
\left.\mu_{\alpha}^{(0)}\right|_{I} ^{ \pm}+\left.z_{\alpha} \varphi^{(0)}\right|_{I} ^{ \pm}=\underset{s}{\mu_{\alpha}^{(0)}}+\underset{s}{z_{\alpha}} \varphi^{(0)}
$$


Using the momentum balance equation (5.27b) and the Poisson equation $(5.27 c)$, we can re-write the jump condition $(5.28 b)$ into

$$
\left.p^{(0)}\right|_{I} ^{+}=\left.p^{(0)}\right|_{I} ^{-}
$$

\subsubsection{Higher order bulk}

The definitions of the boundary layer charge density in (4.7) and of the boundary layer tension in (4.14) read in dimensionless form

$$
\widetilde{q}^{ \pm}= \pm \int_{0}^{ \pm \infty} \widetilde{n}^{\mathrm{F},(0)} d z, \quad \widetilde{\gamma}^{ \pm}= \pm \int_{0}^{ \pm \infty}\left(1+\chi_{ \pm}\right)\left(\partial_{z} \widetilde{\varphi}^{(0)}\right)^{2} \mathrm{~d} z
$$

Integration of $(5.27 c)$ and the matching condition for $\partial_{z} \widetilde{\varphi}^{(0)}$ show that the jump condition $(5.28 c)$ can be written in the form

$$
0=\delta n_{s}^{\mathrm{F},(0)}+\widetilde{q}^{+}+\widetilde{q}^{-} .
$$

From Poisson equations (5.27c) and (5.29c) at leading and higher order, we get

$$
\begin{aligned}
& \widetilde{n}^{\mathrm{F},(0)} \partial_{z} \widetilde{\varphi}^{(1)}+\widetilde{n}^{\mathrm{F},(1)} \partial_{z} \widetilde{\varphi}^{(0)} \\
& \quad=-(1+\chi) \partial_{z}\left(\partial_{z} \widetilde{\varphi}^{(0)} \partial_{z} \widetilde{\varphi}^{(1)}\right)+2 k_{M}^{(0)}(1+\chi)\left(\partial_{z} \widetilde{\varphi}^{(0)}\right)^{2} .
\end{aligned}
$$

Thus, the momentum balance $(5.29 b)$ can be re-written as

$$
\partial_{z} \widetilde{p}^{(1)}-(1+\chi) \partial_{z}\left(\partial_{z} \widetilde{\varphi}^{(0)} \partial_{z} \widetilde{\varphi}^{(1)}\right)=-2 k_{M}^{(0)}(1+\chi)\left(\partial_{z} \widetilde{\varphi}^{(0)}\right)^{2} .
$$

Integration from $z=0$ to $\pm \infty$ yields

$$
\left.p^{(1)}\right|_{I} ^{ \pm}-\left.\left(\widetilde{p}^{(1)}-(1+\chi) \partial_{z} \widetilde{\varphi}^{(0)} \partial_{z} \widetilde{\varphi}^{(1)}\right)\right|_{z=0} ^{ \pm}=\mp 2 k_{M}^{(0)} \widetilde{\gamma}^{ \pm}
$$

where we have used the matching conditions (5.31b), (5.32a) and the bulk equation (5.16). Now, we can write the jump condition $(5.30 b)$ as

$$
\left.p^{(1)}\right|_{I} ^{+}-\left.p^{(1)}\right|_{I} ^{-}=2 k_{M}^{(0)}\left(\delta \gamma_{s}^{(0)}-\widetilde{\gamma}^{+}-\widetilde{\gamma}^{-}\right) .
$$

Finally, due to the constancy of the inner electrochemical potentials, we can relate the electrochemical potentials of the outer expansion to the boundary values at $S$ by

$$
\left.\mu_{\alpha}^{(1)}\right|_{I} ^{ \pm}+\left.z_{\alpha} \varphi^{(1)}\right|_{I} ^{ \pm}=\underset{s}{\mu_{\alpha}^{(1)}}+\underset{\alpha}{z_{\alpha}} \varphi^{(1)}
$$

\section{Mathematical derivation of the Lippmann equation}

In the following derivation of the Lippmann equation only leading order variables are involved. Thus, to simplify the notation, we omit the labelling of the leading order terms 
by the index (0). Recall that within the leading order bulk system the electric potential is constant, and thus the potential difference $U=\varphi^{+}-\varphi^{-}$according to (4.22) is well defined. Moreover, the number densities $n_{\alpha}^{ \pm}$and hence $\mu_{\alpha}^{ \pm}$are constant in $\Omega^{ \pm}$and independent of $U$, i.e.,

$$
\frac{d}{d U} \mu_{\alpha}^{ \pm}=0
$$

Finally, the pressure is constant in $\Omega$ and we denote $p^{r e f}=p$.

The derivation of the Lippmann equation consists of a straightforward calculation of the derivative with respect to $U$ of the interfacial tension $\gamma=\gamma_{s}-\widetilde{\gamma}^{+}-\widetilde{\gamma}^{-}$as defined in (4.23). We start with the term related to the surface stress $\gamma$.

\subsection{Surface contribution}

From the Gibbs-Duhem relation (2.7) right and the global electroneutrality $(4.6 b)$, we infer

$$
\frac{d}{d U} \gamma=-\sum_{\alpha \in \mathcal{M}_{S}} n_{s} \frac{d}{d U} \mu_{s}=-\sum_{\alpha \in \mathcal{M}_{S}} n_{s} \frac{d}{d U}\left(\mu_{s}+z_{\alpha} e_{0} \varphi\right)-\left(\widetilde{q}^{+}+\widetilde{q}^{-}\right) \frac{d}{d U} \varphi_{s} .
$$

First, we consider all contributions to the sum in (6.2) with $\alpha \in \mathcal{M}^{ \pm} \subseteq \mathcal{M}_{S}$. We replace the surface electrochemical potentials by the corresponding bulk quantities according to (4.6a) and use (4.22) and the global electroneutrality (4.6b).

$$
\begin{aligned}
& -\sum_{\alpha \in \mathcal{M}^{ \pm}} n_{s} \frac{d}{d U}\left(\mu_{\alpha}+z_{\alpha} e_{0} \varphi\right) \\
= & -\sum_{\alpha \in \mathcal{M}^{-}} z_{\alpha} e_{0} n_{\alpha} \frac{d}{d U} \varphi^{-}-\sum_{\alpha \in \mathcal{M}^{+}} z_{\alpha} e_{0} n_{s} \frac{d}{d U} \varphi^{+} \\
= & -\sum_{\alpha \in \mathcal{M}^{-}} z_{\alpha} e_{0} n_{\alpha} \frac{d}{d U} \varphi^{-} \\
& \left.+\sum_{\alpha \in \mathcal{M}^{-}} z_{\alpha} e_{0} n_{\alpha}+\sum_{\alpha \in \mathcal{M}_{S} \backslash \mathcal{M}^{ \pm}} z_{\alpha} e_{0} n_{\alpha}+\widetilde{q}^{+}+\widetilde{q}^{-}\right) \frac{d}{d U} \varphi^{+} \\
= & \sum_{\alpha \in \mathcal{M}^{-}} z_{\alpha} e_{0} n_{\alpha}+\left(\widetilde{q}^{+}+\widetilde{q}^{-}\right) \frac{d}{d U} \varphi^{+}+\sum_{\alpha \in \mathcal{M}_{S} \backslash \mathcal{M}^{ \pm}} z_{\alpha} e_{0} n_{\alpha} \frac{d}{d U} \varphi^{+} .
\end{aligned}
$$

Next, we consider the remaining terms in (6.2) from the exclusive surface species. We apply (4.20), reformulate in terms of bulk quantities due to (4.6a) and use (6.1) to get

$$
\begin{aligned}
& -\sum_{\alpha \in \mathcal{M}_{S} \backslash \mathcal{M}^{ \pm}} n_{s} \frac{d}{d U}\left(\mu_{\alpha}+z_{\alpha} e_{0} \varphi\right) \\
= & -\sum_{\alpha \in \mathcal{M}_{s} \backslash \mathcal{M}^{ \pm}} n_{s}\left(\sum_{\beta \in \mathcal{M}^{-}} v_{\beta \alpha} z_{\beta} e_{0} \frac{d}{d U} \varphi^{-}+\sum_{\beta \in \mathcal{M}^{+}} v_{\beta \alpha} z_{\beta} e_{0} \frac{d}{d U} \varphi^{+}\right) .
\end{aligned}
$$


Since $-\varphi^{-}=U-\varphi^{+}$and hence

$$
-\sum_{\beta \in \mathcal{M}^{-}} v_{\beta \alpha} z_{\beta} e_{0} \frac{d}{d U} \varphi^{-}=\sum_{\beta \in \mathcal{M}^{-}} v_{\beta \alpha} z_{\beta} e_{0}-\sum_{\beta \in \mathcal{M}^{-}} v_{\beta \alpha} z_{\beta} e_{0} \frac{d}{d U} \varphi^{+}
$$

and using (2.3a) we get from (6.4)

$$
\begin{aligned}
& -\sum_{\alpha \in \mathcal{M}_{S} \backslash \mathcal{M}^{ \pm}}{ }_{s} n_{\alpha} \frac{d}{d U}\left(\mu_{\alpha}+z_{\alpha} e_{0} \varphi\right) \\
= & \sum_{\alpha \in \mathcal{M}_{S} \backslash \mathcal{M}^{ \pm}} n_{s}\left(\sum_{\beta \in \mathcal{M}^{-}} v_{\beta \alpha} z_{\beta} e_{0}-\sum_{\beta \in \mathcal{M}^{ \pm}} v_{\beta \alpha} z_{\beta} e_{0} \frac{d}{d U} \varphi^{+}\right) \\
= & \sum_{\alpha \in \mathcal{M}_{S} \backslash \mathcal{M}^{ \pm}}\left(\sum_{\beta \in \mathcal{M}^{-}} v_{\beta \alpha} z_{\beta} e_{0} n_{\alpha}-z_{\alpha} e_{0} n_{\alpha} \frac{d}{d U} \varphi^{+}\right) .
\end{aligned}
$$

Combining (6.2), (6.3c), and (6.6), we conclude that the leading order charge contribution of the surface is

$$
\frac{d}{d U} \gamma=\sum_{\alpha \in \mathcal{M}^{-}} z_{\alpha} e_{0} n_{s}+\sum_{\alpha \in \mathcal{M}_{S} \backslash \mathcal{M}^{ \pm}} \sum_{\beta \in \mathcal{M}^{-}} v_{\beta \alpha} z_{\beta} e_{0} n_{s}+\left(\widetilde{q}^{+}+\widetilde{q}^{-}\right) \frac{d}{d U}\left(\varphi^{+}-\varphi_{s}\right) .
$$

\subsection{Boundary layer contributions}

The leading order electrochemical potentials are constant in the layers due to (4.9) and equal to the surface electrochemical potentials due to $(4.6 a)$. Therefore in the boundary layers we have

$$
\widetilde{\mu}_{\alpha}=\mu_{\alpha}^{ \pm}-z_{\alpha} e_{0}\left(\widetilde{\varphi}-\varphi^{ \pm}\right)
$$

From this expression, we conclude that the leading order number densities $\widetilde{n}_{\alpha}$ in the boundary layers can be expressed as a functions of the potential differences $\widetilde{\varphi}-\varphi^{+}$and $\widetilde{\varphi}-\varphi^{-}$, respectively. Further, we conclude from the momentum balance (4.13) and Poisson equation (4.10)

$$
\widetilde{p}-p^{r e f}=\left(1+\chi_{ \pm}\right) \varepsilon_{0}\left(\partial_{z} \widetilde{\varphi}\right)^{2}
$$

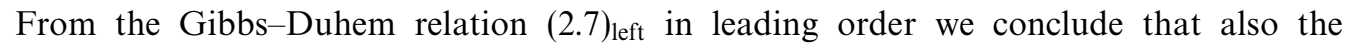
pressure $\widetilde{p}$ is a function of the potential differences $\widetilde{\varphi}-\varphi^{ \pm}$. Assuming monotonicity of $\widetilde{\varphi}$ in the boundary layers, we get a differential equation for $\widetilde{\varphi}$ of the form

$$
\left(1+\chi_{ \pm}\right) \varepsilon_{0} \partial_{z} \widetilde{\varphi}=F^{ \pm}\left(\widetilde{\varphi}-\varphi^{ \pm}\right)
$$


where the functions $F^{ \pm}$are defined as $F^{ \pm}:= \pm \operatorname{sgn}\left(\varphi^{ \pm}-\varphi\right) \sqrt{\left(1+\chi_{ \pm}\right) \varepsilon_{0}\left(\widetilde{p}-p^{r e f}\right)}$. Then, we can re-write the boundary layer tension $\widetilde{\gamma}^{ \pm}$as

$$
\widetilde{\gamma}^{ \pm}= \pm \int_{0}^{ \pm \infty}\left(1+\chi_{ \pm}\right) \varepsilon_{0}\left(\partial_{z} \widetilde{\varphi}\right)^{2} \mathrm{~d} z= \pm \int_{\widetilde{\varphi}(0)}^{\widetilde{\varphi}( \pm \infty)} F\left(\widetilde{\varphi}-\varphi^{ \pm}\right) d \widetilde{\varphi}= \pm \int_{\varphi}^{\varphi^{ \pm}} F^{ \pm}\left(\widetilde{\varphi}-\varphi^{ \pm}\right) d \widetilde{\varphi}
$$

We define $U^{ \pm}:=\varphi^{ \pm}-\varphi_{s}$ and differentiate (6.11) with respect to $U^{ \pm}$to get

$$
\frac{d}{d U^{ \pm}} \widetilde{\gamma}^{ \pm}= \pm \frac{d}{d U^{ \pm}} \int_{-U^{ \pm}}^{0} F^{ \pm}(\widetilde{\varphi}) d \widetilde{\varphi}= \pm F^{ \pm}\left(-U^{ \pm}\right)= \pm\left(1+\chi_{ \pm}\right) \varepsilon_{0} \partial_{z} \widetilde{\varphi}(0)
$$

We use the Poisson equation (4.10) for the leading order to conclude

$$
\pm\left(1+\chi_{ \pm}\right) \varepsilon_{0} \partial_{z} \widetilde{\varphi}(0)=\mp \int_{0}^{ \pm \infty}\left(1+\chi_{ \pm}\right) \varepsilon_{0} \partial_{z z} \widetilde{\varphi} \mathrm{d} z= \pm \int_{0}^{ \pm \infty} \widetilde{n}^{\mathrm{F}} \mathrm{d} z=\widetilde{q}^{ \pm} .
$$

Since $U=U^{+}-U^{-}$, we have $\frac{d U^{-}}{d U}=\frac{d U^{+}}{d U}-1$ and deduce

$$
\begin{aligned}
\frac{d}{d U}\left(\widetilde{\gamma}^{+}+\widetilde{\gamma}^{-}\right) & =\frac{d U^{+}}{d U} \frac{d}{d U^{+}} \widetilde{\gamma}^{+}+\frac{d U^{-}}{d U} \frac{d}{d U^{-}} \widetilde{\gamma}^{-} \\
& =\frac{d U^{+}}{d U} \widetilde{q}^{+}+\left(\frac{d U^{+}}{d U}-1\right) \widetilde{q}^{-} \\
& =-\widetilde{q}^{-}+\left(\widetilde{q}^{+}+\widetilde{q}^{-}\right) \frac{d U^{+}}{d U} .
\end{aligned}
$$

Putting (6.7) and (6.14) together, we finally conclude

$$
\frac{d}{d U}\left(\gamma-\widetilde{\gamma}_{s}^{+}-\widetilde{\gamma}^{-}\right)=\widetilde{q}^{-}+\sum_{\alpha \in \mathcal{M}^{-}} z_{\alpha} e_{0} n_{\alpha}+\sum_{\alpha \in \mathcal{M}_{s} \backslash \mathcal{M}^{ \pm}} \sum_{\beta \in \mathcal{M}^{-}} v_{\beta \alpha} z_{\beta} e_{0} n_{s} .
$$

With the definition (4.21) of the double layer charge density, we thus get the Lippmann equation (4.18).

\section{Material model for the metal-electrolyte interface}

Next, we want to apply the general Lippmann equation resulting from the asymptotic analysis to various metal|aqueous electrolyte interfaces. For this reason, we have to specify a material model for the metal-electrolyte interface. In particular, we have to specify the chemical potentials for both bulk phases as well as for the surface and the considered reactions in the electrolyte bulk and on the surface. We apply the material model that is derived and analyzed in detail in [31]. It is briefly summarized below together with some useful representation formulas for the surface mole fractions, the interfacial tension and electric charge as function of potential difference between metal and electrolyte. 


\section{The model equations and simplified notation}

To study the electrocapilarity curves it is only necessary to consider the leading order equations of boundary layer and the surface equations (4.6)-(4.14). Thus, we can omit the labelling of the leading order terms with (0) for simplicity of notation. The bulk quantities in the leading order are constant and serve only as boundary values for the boundary layer model. We highlight the bulk quantities by the index $M$ and $E$ for the metal and the electrolyte, respectively. Due to this notation a labelling of the boundary layer quantities with tilde is not necessary anymore. Also, the indication of the boundary layer charge density and the boundary layer tension by tilde is omitted and replaced by $Q_{\mathrm{BL}}^{\mathrm{M}, \mathrm{E}}=\widetilde{q}^{ \pm}$ and $\gamma_{\mathrm{BL}}^{\mathrm{M}, \mathrm{E}}=\widetilde{\gamma}^{ \pm}$, respectively. By convention, we let the metal occupy the domain $\Omega^{+}=\Omega^{\mathrm{M}}$ and accordingly, the electrolyte occupies the domain $\Omega^{-}=\Omega^{\mathrm{E}}$. Further, we denote the potential difference in the leading order between metal-surface and electrolyte-surface as

$$
U^{\mathrm{M}}=\varphi^{\mathrm{M}}-\varphi_{s} \quad \text { and } \quad U^{\mathrm{E}}=\varphi_{s}-\varphi^{\mathrm{E}}
$$

\subsection{Specific material model}

\subsubsection{Metal}

The metal is modelled as a binary mixture of metal ions $\mathrm{M}$ and valence electrons $\mathrm{e}^{-}$, i.e., the index set for the metal is $\mathcal{M}_{\mathrm{E}}=\left\{\mathrm{M}, \mathrm{e}^{-}\right\}$. The respective mole densities are denoted by $n_{M}$ and $n_{e}$. We assume the metal to be incompressible and consider the electrons to be point charges, leading to the incompressibility constraint $n_{M} v_{M}^{r e f}=1$, where $v_{M}^{r e f}$ denotes the partial volume of the metal ion. For the chemical potentials, we have

$$
\mu_{M}=\psi_{M}^{r e f}+v_{M}^{r e f} p_{M} \quad \text { and } \quad \mu_{e}=\left(\frac{3}{8 \pi}\right)^{2 / 3} \frac{h^{2}}{2 m_{e}} n_{e}^{2 / 3}
$$

where $\mu_{e}$ is equal to the Fermi level of the considered metal. Note that $p_{M}$ in (7.2) is the metal ion partial pressure that is related to the total material pressure $p$ via

$$
p=p_{e}+p_{M} \quad \text { and } \quad p_{e}=\frac{2}{5}\left(\frac{3}{8 \pi}\right)^{2 / 3} \frac{h^{2}}{2 m_{e}} n_{e}^{5 / 3} .
$$

\subsubsection{Electrolyte}

We consider the electrolyte as incompressible liquid mixture of a solvent, undissociated species and ionic species. The ionic species are considered as solvated ions, i.e., they are composed of a centre ion and a surrounding shell of several bounded solvent molecules, cf. Figure 3. The index set of the electrolytic species is denoted by $\mathcal{M}_{\mathrm{E}}$. For each constituent, $v_{\alpha}^{r e f}$ is the specific volume, and $\kappa_{\alpha}$ is the solvation shell number. Since we consider a mixture of solvated ions, the partial volumes of the ionic species are much larger than 


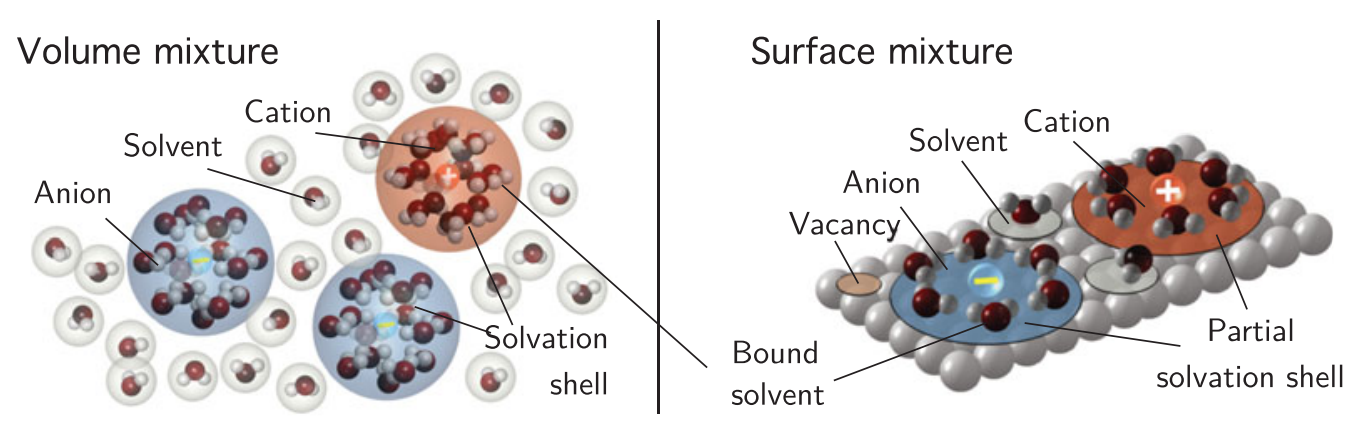

FIGURE 3. Sketch of the mixture constituents in the volume and on the surface. Anions and cations consists of a centre ion and a surrounding solvation shell of bounded and oriented solvent molecules. In addition, there may be free solvent molecules and unoccupied sites on the surface.

the partial volume of the solvent. The mole fractions $y_{\alpha}$ of the constituents are defined as

$$
y_{\alpha}=\frac{n_{\alpha}}{n} \quad \text { with } \quad n=\sum_{\beta \in \mathcal{M}_{\mathrm{E}}} n_{\beta} .
$$

The incompressibility of the electrolyte states the condition

$$
n \sum_{\alpha \in \mathcal{M}_{\mathrm{E}}} v_{\alpha}^{r e f} y_{\alpha}=1
$$

The chemical potentials of the electrolytic constituents in the incompressible limit are

$$
\mu_{\alpha}=g_{\alpha}^{r e f}+v_{\alpha}^{r e f}\left(p-p^{r e f}\right)+k_{B} T \ln \left(y_{\alpha}\right), \quad \alpha \in \mathcal{M}_{\mathrm{E}},
$$

with reference Gibbs free energy $g_{\alpha}^{r e f}=\psi_{\alpha}^{r e f}+v_{\alpha}^{r e f} p^{r e f}$. In contrast to the standard literature, cf. $[3,8,37]$, the chemical potentials contain a pressure dependent contribution that is of crucial importance inside the boundary layers [31]. It is possible to reformulate (7.6) in such a way that the pressure contribution yields an activity coefficient different from unity in the logarithmic term, as proposed in [21] for numerical purpose.

\subsubsection{Surface}

The surface $S$ is considered as mixture of the surface metal ions, surface electrons, electrolytic adsorbates and surface reaction products, with respective surface number densities $n_{\alpha}$. Note that we consider on the surface also a solvation effect, whereby each adsorbed ion binds $\kappa_{\alpha}$ solvent molecules. The index set of surface constituents is denoted by $\mathcal{M}_{S} \supseteq \mathcal{M}_{\mathrm{E}, \mathrm{M}}$, where $\mathcal{M}_{\mathrm{E}, \mathrm{M}}=\mathcal{M}_{\mathrm{E}} \cup \mathcal{M}_{\mathrm{M}}$.

Analogously to the metal volume, we have an incompressibility constraint on the surface stating $a_{M}^{r e f} n_{s}=1$, where $a_{M}^{r e f}$ is the partial area of surface metal ions. On the electrolyte side, we have to account for adsorption from the volume. Each surface metal ion offers 
$\omega_{M}$ adsorption sites. Thus, the number density of possible adsorption sides is

$$
\underset{s}{n}=\omega_{M} n_{M}
$$

Since the surface does not need to be completely covered with adsorbates, we introduce a mole density of surface vacancies via

$$
n_{V}=\omega_{M} n_{s}-\sum_{\alpha \in \mathcal{M}_{S} \backslash \mathcal{M}_{M}} \omega_{\alpha} n_{\alpha} .
$$

Here, $\omega_{\alpha}$ denotes the adsorption sites occupied by each species of the adsorbates. The specific areas of vacancies and adsorbates are given by the adsorption sites, i.e.,

$$
a_{V}^{r e f}=\frac{1}{\omega_{M}} a_{M}^{r e f} \quad \text { and } \quad a_{\alpha}^{r e f}=\frac{\omega_{\alpha}}{\omega_{M}} a_{M}^{r e f}
$$

We introduce the surface fraction of vacancies and of adsorbates as

$$
y_{S}=\frac{n_{V}}{n_{s}} \quad \text { and } \quad y_{s}=\frac{n_{\alpha}}{n} .
$$

The chemical potentials of the adsorbates are

$$
\mu_{\alpha}=\psi_{s}^{r e f}+k_{B} T \ln y_{s}-\omega_{\alpha} k_{B} T \ln y_{S}
$$

The chemical potential of the metal ions is

$$
\underset{s}{\mu_{M}}=\psi_{s}^{r e f}+\omega_{M} k_{B} T \ln y_{s}-a_{M}^{r e f} \gamma_{s}
$$

For the electrons we assume a constant surface chemical potential, i.e.,

$$
\mu_{s}=\underset{s}{\mu_{e}^{\mathrm{M}}}
$$

We are not aware of a comparable surface model in the standard literature.

\subsubsection{Chemical reactions}

In order to describe a wide range of metal-electrolyte-interfaces, we consider several chemical reactions in the volume and on the surface.

In the electrolyte several volume reactions may occur, which account for the dissociation of acids, the dissolution of salts, also the self-ionization of the solvent. Each volume reaction can easily be taken into account because under the given equilibrium conditions the reaction impose a restriction on the bulk values of the chemical potentials of the electrolyte species.

In general, all electrolytic constituents can adsorb on the surface. The adsorption of the ionic species is usually followed by a reorganization of the solvation shell. This process can be described as a reaction in which where the adsorbates loose a part of their solvation shell. Moreover, the adsorbates can be involved in further surface reactions 
where electrons and the metal ions are involved. We assume that the reaction products are exclusive surface constituents, such that there is no charge transfer between the two bulk phases. Also these surface reactions can be accompanied by further changes of the solvation shell of the charge species. All those surface reactions can be represented in the form (4.19).

\subsection{Implications of the material model}

\subsubsection{Constant potential difference $U^{\mathrm{M}}$}

The potential drop $U^{\mathrm{M}}$ in the metal is determined by the adsorption equilibrium of the electrons $(4.6 a)$ on the metal surface as

$$
U^{\mathrm{M}}=\frac{1}{e_{0}}\left(\mu_{e}^{\mathrm{M}}-\mu_{s}^{\mathrm{M}}\right) .
$$

Due to the constitutive model for the metal and the properties of the leading order quantities in the bulk the chemical potentials $\mu_{e}^{\mathrm{M}}$ as well as $\mu_{s}^{\mathrm{M}}$ are constant. Therefore, the potential difference $U^{\mathrm{M}}$ is independent of the applied potential difference $U$ across the complete double layer, and depends only on the constant material parameters $\mu_{e}^{\mathrm{M}}$ and $\mu_{e}^{\mathrm{M}}$.

\subsubsection{Decomposition of interfacial tension}

The constitutive function (7.11b) for the metal ions and the adsorption equilibrium (4.6a) imply

$$
a_{M}^{r e f} \underset{s}{\gamma}=\psi_{s}^{r e f}+\omega_{M} k_{B} T \ln {\underset{s}{y}}_{V}-\mu_{M}^{\mathrm{M}}-z_{M} e_{0} U^{\mathrm{M}}
$$

As a direct consequence of the constant potential difference $U^{\mathrm{M}}$ according to (7.12), only the term containing $y_{V}$ can depend on $U^{\mathrm{E}}$ and all constant terms in (7.13) are related to the metal. This motivates the splitting of the surface tension as

$$
\gamma=\gamma_{s}^{\mathrm{M}}-\gamma_{s}^{\mathrm{E}}
$$

with

$$
\underset{s}{\gamma^{\mathrm{M}}}:=\frac{1}{a_{M}^{r e f}}\left(\psi_{s}^{r e f}-\mu_{M}^{\mathrm{M}}-z_{M} e_{0} U^{\mathrm{M}}\right) \quad \text { and } \quad \gamma_{s}^{\mathrm{E}}:=-\frac{\omega_{M}}{a_{M}^{\text {ref }}} k_{B} T \ln \ln _{V}
$$

By this definition $\gamma_{s}^{\mathrm{M}}$ is constant and and $\gamma_{S}^{\mathrm{E}}$ is always non-negative and vanishes in the case of of a clean surface, implying $\gamma_{s}=\gamma_{s}^{\mathrm{M}}$ for $y_{s}=1$. With the boundary layer tensions $\gamma_{\mathrm{BL}}^{\mathrm{E}}$ and $\gamma_{\mathrm{BL}}^{\mathrm{M}}$, we introduce the metallic and electrolytic interfacial tension as

$$
\gamma^{\mathrm{M}}:=\gamma_{s}^{\mathrm{M}}-\gamma_{\mathrm{BL}}^{\mathrm{M}} \quad \text { and } \quad \gamma^{\mathrm{E}}:=\gamma_{s}^{\mathrm{E}}+\gamma_{\mathrm{BL}}^{\mathrm{E}}
$$


Then, we can split the interfacial tension in a similar fashion to $(7.14 a)$ as

$$
\gamma=\gamma^{\mathrm{M}}-\gamma^{\mathrm{E}}
$$

By definition the boundary layer tensions $\gamma_{\mathrm{BL}}^{\mathrm{E}}$ and $\gamma_{\mathrm{BL}}^{\mathrm{M}}$ are non-negative and $\gamma_{\mathrm{BL}}^{\mathrm{M}}$ is independent of $U^{\mathrm{E}}$ as a direct consequence of the constant potential difference $U^{\mathrm{M}}$. Thus, metallic interfacial tension $\gamma^{\mathrm{M}}$ also is constant and the electrolytic interfacial tension $\gamma^{\mathrm{E}}$ is non-negative. We conclude, that the electrolytic interfacial tension always lowers the interfacial tension due to adsorption and charge accumulation in the electrolytic boundary layer.

\subsubsection{Current-Charge relation}

From a mathematical point of view, the system (4.6)-(4.14) is identical to the one dimensional system of [31] for a planar metal-electrolyte interface, and we also apply the same material model here. Moreover, we see that the double layer charge density (4.21) is exactly the same electric charge density $Q$, which we have already deduced in [31] in the context of the charge-current relation

$$
I=\frac{d Q}{d t}
$$

where $I$ is the current per surface area which flows into the double layer from the metal side. Thus, it is possible to establish the same current-charge relation here, the derivation is given in the Appendix B. The current-charge relation allows to measure the double layer charge by an experiment independent from the Lippmann equation, for instance the classical dropping mercury electrode [18] and thereby enables an experimental confirmation of the Lippmann equation.

\subsection{Representation formulas for the metal-electrolyte interface}

Several useful representation formulas for the metal-electrolyte interface can be derived in the same way like in [31]. They are summarized here because the computations of Section 8 are based on them.

\subsubsection{Pressure $p$ as function of potential}

The definitions of the mole fractions requires in the electrolyte boundary layer the side condition $1=\sum_{\alpha \in \mathcal{M}_{\mathrm{E}}} y_{\alpha}$. Thus, the boundary layer equations (4.9) imply the algebraic equation between the pressure and the electrostatic potential

$$
\sum_{\alpha \in \mathcal{M}_{\mathrm{E}}} y_{\alpha}^{\mathrm{E}} \exp \left(-\frac{z_{\alpha} e_{0}}{k_{B} T}\left(\varphi-\varphi^{\mathrm{E}}\right)-\frac{v_{\alpha}^{r e f}}{k_{B} T}\left(p-p^{\mathrm{E}}\right)\right)=1 .
$$

In particular, the pressure $p$ in electrolytic boundary layer can be expressed as a function of the potential difference $\varphi-\varphi^{\mathrm{E}}$, i.e. $p=p\left(\varphi-\varphi^{\mathrm{E}}\right)$. 


\subsubsection{Boundary layer tension $\gamma_{B L}^{\mathrm{E}}$}

Further, we have a representation of the electrolytic boundary layer tension

$$
\gamma_{\mathrm{BL}}^{\mathrm{E}}=\int_{0}^{U^{\mathrm{E}}} \sqrt{2 \varepsilon_{0}(1+\chi)\left(p(u)-p^{\mathrm{E}}\right)} d u
$$

where $p(u)$ is the material pressure according to (7.18) with $\varphi=\varphi^{\mathrm{E}}+u$.

\subsubsection{Surface mole fractions $y_{\alpha}$}

The surface mole fraction $y_{V}$ of the vacancies can be expressed as a function of the electrolytic surface tension,

$$
y_{S}=\exp \left(-\frac{a_{V}^{r e f}}{k_{B} T} \gamma_{s}^{\mathrm{E}}\right) .
$$

The mole fraction $y_{\alpha}$ for the electrolytic adsorbates, i.e., $\alpha \in \mathcal{M}_{\mathrm{E}}$, we have the representations

$$
y_{\alpha}=y_{\alpha}^{\mathrm{E}} \exp \left(-\frac{\Delta \widetilde{g}_{\alpha}}{k_{B} T}-\frac{e_{0}}{k_{B} T} z_{\alpha} U^{\mathrm{E}}-\frac{a_{\alpha}^{r e f}}{k_{B} T} \gamma_{s}^{\mathrm{E}}\right),
$$

with the corresponding Gibbs energies defined by

$$
\Delta \widetilde{\mathrm{g}}_{\alpha}=\psi_{\alpha}^{r e f}-\left(\psi_{\alpha}^{r e f}+v_{\alpha}^{r e f} p^{\mathrm{E}}\right)
$$

For the surface reaction products, i.e., $\mathrm{A}_{\beta}$ with $\beta \in \mathcal{M}_{S} \backslash \mathcal{M}_{\mathrm{E}, \mathrm{M}}$, we obtain from the general equilibrium conditions (4.20) and (4.6a) of the net reactions (4.19) the representation

$$
y_{s}=\prod_{\alpha \in \mathcal{M}_{\mathrm{E}}}\left(y_{\alpha}^{\mathrm{E}}\right)^{v_{\alpha \beta}} \exp \left(-\frac{\Delta \widetilde{\mathrm{g}}_{\beta}}{k_{B} T}-\frac{e_{0}}{k_{B} T}\left(\sum_{\delta \in \mathcal{M}_{\mathrm{E}}} v_{\delta \beta} z_{\delta}\right) U^{\mathrm{E}}-\frac{a_{\beta}^{r e f}}{k_{B} T} \gamma_{s}^{\mathrm{E}}\right),
$$

where we again define Gibbs energies as

$$
\Delta \widetilde{g}_{\beta}=\psi_{s}^{r e f}-\sum_{\alpha \in \mathcal{M}_{M}} v_{\alpha \beta}\left(\mu_{\alpha}^{\mathrm{M}}+e_{0} z_{\alpha} U^{\mathrm{M}}\right)-\sum_{\alpha \in \mathcal{M}_{\mathrm{M}}} v_{\alpha \beta}\left(\psi_{\alpha}^{r e f}+v_{\alpha}^{r e f} p^{\mathrm{E}}\right) .
$$

From these representation formulas, we conclude that the Gibbs energies control the amount of adsorbates and reaction products on the surface. In case of charged species, the Gibbs energies refer to the whole solvated ion, which includes the Gibbs energy of the centre ion as well as the Gibbs energy of the solvent molecules. To separate the different contributions, we introduce the decomposition

$$
\Delta \widetilde{g}_{\beta}=\Delta g_{\beta}+\kappa_{\beta} \Delta g_{0} .
$$


The index 0 refers to the solvent with the adsorption energy $\Delta g_{0}$ according to $(7.21 b)$. Now, $\Delta g_{\beta}$ corresponds to the adsorption energy of a single molecule, and can be expected in the order of $1 \mathrm{eV}$.

\subsubsection{Relation between $\gamma_{s}^{\mathrm{E}}$ and $U^{\mathrm{E}}$}

The above representations and the definition (7.10) of the surface mole fractions yield an algebraic equation which determines $\gamma_{s}^{\mathrm{E}}$ as a function of $U^{\mathrm{E}}$, viz.,

$$
y_{S}+\sum_{\alpha \in \mathcal{M}_{S} \backslash \mathcal{M}_{M}} y_{\alpha}=1
$$

\subsubsection{Electrolytic boundary layer charge density $Q_{B L}^{\mathrm{E}}$}

It is remarkable that it is possible to determine the boundary layer charge density $Q_{\mathrm{BL}}^{\mathrm{E}}$ without needing to spatially resolve the boundary layer equations (4.9)-(4.13), i.e.,

$$
Q_{\mathrm{BL}}^{\mathrm{E}}=\operatorname{sgn}\left(U^{\mathrm{E}}\right) \sqrt{2 \varepsilon_{0}(1+\chi)\left(p\left(U^{\mathrm{E}}\right)-p^{\mathrm{E}}\right)} .
$$

where $p\left(U^{\mathrm{E}}\right)$ is the material pressure according to (7.18).

\subsubsection{Surface charge density}

Once all surface mole fractions are determined according to (7.20)-(7.22), we get for the surface charge density

$$
Q_{s}^{\mathrm{E}}=-\frac{\sum_{\left(\alpha \in \mathcal{M}_{\mathrm{E}}\right)} z_{\alpha} e_{0} y_{\alpha}+\sum_{\left(\alpha \in \mathcal{M}_{\mathrm{E}}\right)} \sum_{\left(\beta \in \mathcal{M}_{S} \backslash \mathcal{M}_{\mathrm{E}, \mathrm{M}}\right)} v_{\alpha \beta} z_{\alpha} e_{0} y_{\beta}}{a_{V}^{r e f} y_{S}+\sum_{\left(\alpha \in \mathcal{M}_{S} \backslash \mathcal{M}_{\mathrm{M}}\right)} a_{\alpha}^{r e f} y_{s}} .
$$

Thus, the surface charge density itself is a function of the potential difference $U^{\mathrm{E}}$ and the surface tension $\gamma$.

\section{Electrocapillarity of the $\mathrm{Hg} \mid$ aqueous electrolyte interface}

In this section, we relate our model to experimental data for several representative examples of the $\mathrm{Hg} \mid$ aqueous electrolyte interface like the ones given in Figure 1a. In order to compute the electrocapillarity curves, we determine for each given $U^{\mathrm{E}}$ :

- $\gamma_{\mathrm{BL}}^{\mathrm{E}}$ by numerically integrating (7.19), where in the integrand $p(u)$ is the iterative numerical solution of (7.18),

- $\gamma^{\mathrm{E}}$ by iterative numerical solution of (7.24), where $y_{V}$ and $y_{\alpha}$ for $\alpha \in \mathcal{M}_{S} \backslash \mathcal{M}_{\mathrm{M}}$ are given by (7.20), (7.21), and (7.22). 
The behaviour of the electrocapillarity curves is determined by several parameters of the thermodynamic model. The bulk parameters,

bulk particle densities $n_{\alpha}^{\mathrm{E}}, \quad$ bulk pressure $p^{\mathrm{E}}, \quad$ temperature $T$,

can be adjusted in the experimental setup. In Section 8.1, we discuss briefly the material parameters of the $\mathrm{Hg}$ surface and the solvent $\mathrm{H}_{2} \mathrm{O}$, i.e.,

metal interfacial tension $\gamma^{\mathrm{M}}$, adsorption site per surface metal ion $\omega_{M}$, adsorption energy $\Delta g_{\mathrm{H}_{2} \mathrm{O}}$.

Subsequently, we discuss the dependency of the electrocapillarity curve on the material parameters of the ionic constituents. In Section 8.2, we examine the influence of the remaining material parameters:

$\begin{array}{ll}\text { charge numbers } z_{\alpha}, & \text { dielectric susceptibility } \chi, \\ \text { solvation number } \kappa_{\alpha}, & \text { adsorption energy } \Delta g_{\alpha}, \\ \text { specific volumes } v_{\alpha}^{r e f}, & \text { specific areas } a_{\alpha}^{r e f} .\end{array}$

Although our model allows general ionic species, we restrict our considerations on monovalent salts in order to avoid subtle discussion of questions related to the dissociation degree and the specific volume of a multivalent ion. In the following, the bulk salt concentrations $n_{\alpha}^{\mathrm{E}}$ are set in such a way that the electrolytes represents a $0.1 \mathrm{M}$ solution, if not stated otherwise, and we fix

$$
p^{\mathrm{E}}=10^{5} \mathrm{~Pa} \quad \text { and } \quad T=298 \mathrm{~K}
$$

In the standard experimental three electrode setup, the potential difference between the metal and some reference electrode $\mathrm{R}$ is measured. Therefore, we have to express the the potential difference $U=U^{\mathrm{M}}+U^{\mathrm{E}}$ between the metal and electrolyte as a function of the measured cell potential $E$ between metal and reference electrode. If we assume for the reference electrode the same material model like for the metal, then we have according to Appendix A that

$$
E=U^{\mathrm{E}}+U^{\mathrm{R}}
$$

where $U^{\mathrm{R}}$ denotes a (constant) reference voltage. For simplicity, we set

$$
U^{\mathrm{R}}=0
$$




\subsection{Parameters of solvent and metal surface}

\subsubsection{Specific volume and area of $\mathrm{H}_{2} \mathrm{O}$ and $\mathrm{Hg}$}

The specific volume of the solvent $\mathrm{H}_{2} \mathrm{O}$ and of the metal $\mathrm{Hg}$ can be determined from the mass densities of the pure substances as

$$
v_{\mathrm{H}_{2} \mathrm{O}}^{r e f}=\frac{1}{55.5} \frac{\mathrm{L}}{\mathrm{mol}} \quad \text { and } \quad v_{\mathrm{Hg}}^{r e f}=\frac{1}{67.52} \frac{\mathrm{L}}{\mathrm{mol}} .
$$

We compute the specific areas of $\mathrm{H}_{2} \mathrm{O}$ and $\mathrm{Hg}$ from the simple relation

$$
a_{\alpha}^{r e f}=\frac{2}{\sqrt{3}}\left(2 r_{\alpha}\right)^{2} \quad \text { with } \quad r_{\alpha}=\sqrt[3]{\frac{3 \pi}{4} v_{\alpha}^{r e f}},
$$

which corresponds to a layer of densely packed spheres. The values for $\mathrm{H}_{2} \mathrm{O}$ and $\mathrm{Hg}$ are determined as

$$
a_{\mathrm{H}_{2} \mathrm{O}}^{r e f}=10.33 \cdot 10^{8} \frac{\mathrm{cm}^{2}}{\mathrm{~mol}} \quad \text { and } \quad a_{\mathrm{Hg}}^{r e f}=9.05 \cdot 10^{8} \frac{\mathrm{cm}^{2}}{\mathrm{~mol}} .
$$

Because the specific areas of the water molecules and mercury atoms are quite similar, we assume that each mercury atom offers one adsorption site, i.e.,

$$
\omega_{\mathrm{Hg}}=1 \text {. }
$$

\subsubsection{Interfacial tension $\gamma^{\mathrm{M}}$}

We seek to determine $\gamma^{\mathrm{M}}$ from an experiment that is independent of the actual metal/aqueous electrolyte interface. Therefore, we consider the interface between $\mathrm{Hg}$ and some inert gas and assume also in this setup a material model analogous to Section 7, such that the decomposition $\gamma=\gamma^{\mathrm{M}}-\gamma^{\text {gas }}$ holds true. As the gas phases consist solely of uncharged constituents, there is no boundary layer contribution $\gamma_{\mathrm{BL}}^{\text {gas }}$ to $\gamma^{\text {gas }}$. Additionally, if the gas does not adsorb on the metal (i.e., an inert gas like $\mathrm{N}_{2}$ or Ar), then $\gamma_{s}^{\text {gas }}=0$ and we can read of $\gamma^{\mathrm{M}}$ from an independent measurement of $\gamma^{\mathrm{Hg} \mid \mathrm{gas}}$. With the value [30] $\gamma^{\mathrm{Hg} \mid \text { gas }}=485.5 \cdot 10^{-3} \frac{\mathrm{N}}{\mathrm{m}}$ for the $\mathrm{Hg} \mid$ gas interface at $20^{\circ} \mathrm{C}$, we get

$$
\gamma^{\mathrm{M}}=\gamma^{\mathrm{Hg} \mid \mathrm{gas}}=486.5 \cdot 10^{-3} \frac{\mathrm{N}}{\mathrm{m}} .
$$

\subsubsection{Adsorption energy of the solvent}

Consider the interface between a metal $M$ and pure water, without any additive salt. Then, (7.24) and the representations formulas (7.20) and (7.21) reduce to

$$
\begin{aligned}
& y_{V}+y_{s} y_{\mathrm{H}_{2} \mathrm{O}}=1 \quad \text { with } \quad y_{V}=\exp \left(-\frac{a_{\mathrm{Hg}}^{r e f}}{\omega_{\mathrm{Hg}} k_{B} T} \gamma_{s}^{\mathrm{H}_{2} \mathrm{O}}\right) \\
& \text { and } \quad y_{s} \mathrm{H}_{2} \mathrm{O}=\exp \left(-\frac{\Delta g_{\mathrm{H}_{2} \mathrm{O}}}{k_{B} T}-\frac{a_{\mathrm{H}_{2} \mathrm{O}}^{r e f}}{k_{B} T} \gamma_{s}^{\mathrm{H}_{2} \mathrm{O}}\right) \text {, }
\end{aligned}
$$


Given a value for $\gamma_{s}^{\mathrm{H}_{2} \mathrm{O}}$ from independent experiment, we can thus determine the corresponding solvent adsorption energy $\Delta g_{\mathrm{H}_{2} \mathrm{O}}$. Therefore, we consider the $\mathrm{H}_{2} \mathrm{O} \mid$ gas interface and assume that there also holds the decomposition $\gamma^{\mathrm{H}_{2} \mathrm{O}} \mid$ gas $=\gamma^{\mathrm{H}_{2} \mathrm{O}}-\gamma^{\text {gas }}$ and $\gamma^{\text {gas }}=0$ for a non-adsorbing gas for the same reasons as above. Similar to the gas phase, the boundary layer contribution $\gamma_{\mathrm{BL}}^{\mathrm{H}_{2} \mathrm{O}}$ of water vanishes. Using the same database of Jaspers [30] for the $\mathrm{H}_{2} \mathrm{O} \mid$ gas interface at $20^{\circ} \mathrm{C}$, we then get $\gamma_{\mathrm{s}}^{\mathrm{H}_{2} \mathrm{O}}=\gamma^{\mathrm{H}_{2} \mathrm{O}}=\gamma^{\mathrm{H}_{2} \mathrm{O} \text { gas }}=72.14 \cdot 10^{-3} \frac{\mathrm{N}}{\mathrm{m}}$. As a further implication, we can determine $\gamma^{\mathrm{Hg} \mid \mathrm{H}_{2} \mathrm{O}}=\gamma^{\mathrm{Hg} \mid \text { gas }}-\gamma^{\mathrm{H}_{2} \mathrm{O} \mid \mathrm{gas}}=414.36 \cdot 10^{-3} \frac{\mathrm{N}}{\mathrm{m}}$, a value that coincides well with direct measurements of the $\mathrm{Hg} \mid \mathrm{H}_{2} \mathrm{O}$ interface with [1] $\gamma_{\text {measured }}^{\mathrm{Hg} \mid \mathrm{H}_{2} \mathrm{O}} \approx 415 \cdot 10^{-3} \frac{\mathrm{N}}{\mathrm{m}}$ at $20^{\circ} \mathrm{C}$, which subsequently justifies the assumptions above. We can thus choose $\Delta g_{\mathrm{H}_{2} \mathrm{O}}$ such that $\gamma_{s}^{\mathrm{H}_{2} \mathrm{O}}=72.14 \cdot 10^{-3} \frac{\mathrm{N}}{\mathrm{m}}$, i.e., numerical solution of (8.9) yields

$$
\Delta g_{\mathrm{H}_{2} \mathrm{O}}=-0.0735 \mathrm{eV} \text {. }
$$

\subsection{Non-adsorbing monovalent salt}

Consider some monovalent salt $\mathrm{AC}$ which completely dissociates in anions $\mathrm{A}^{-}$and cations $\mathrm{C}^{+}$and that does not adsorb on the $\mathrm{Hg}$ surface. Thus, only the boundary layer charge density $Q_{\mathrm{BL}}^{\mathrm{E}}$ can cause a potential dependent contribution $\gamma_{\mathrm{BL}}^{\mathrm{E}}$ to the interfacial tension $\gamma$. The amount of charge that can be stored in the layer only depends on the bulk concentration of the salt as well as on parameters $\chi$ and $v_{\alpha}^{r e f}$. Regardless of all possible parameter variation, at the potential of zero charge, i.e., for $E=0$, always the same maximal value of $\gamma$ is attained. To effectively prevent adsorption, we set in the calculations

$$
\Delta g_{\alpha}=+1 \mathrm{eV} \text { for non-adsorbing species. }
$$

\subsubsection{Specific volume}

Due to the incompressibility of the electrolyte, the specific volume $v_{\alpha}^{r e f}$ limits the maximal charge that can be stored per unit volume. We assume $v_{\mathrm{A}^{-}}^{r e f}=v_{\mathrm{C}^{+}}^{r e f}$ such that the electrocapillarity curves are symmetric w.r.t. $E=0$. Then, Figure 4 a shows that by increasing $v_{\alpha}^{\text {ref }}$ the electrocapillarity curves widen up, i.e., the slopes that are related to $Q_{\mathrm{BL}}^{\mathrm{E}}$ decrease. Since we consider the ionic species $\mathrm{A}^{-}$and $\mathrm{C}^{+}$as solvated ions consisting of a charged centre ion and $\kappa_{\alpha}$ solvent molecules in the solvation shell, the simplest choice to determine the specific volume of a solvated ion is

$$
v_{\alpha}^{r e f}=\left(1+\kappa_{\alpha}\right) \cdot v_{0}^{r e f},
$$

where $v_{0}^{r e f}$ is the specific volume of the solvent. For mono-valent ions, we proceed with

$$
v_{\alpha}^{r e f}=45 \cdot v_{0}^{r e f}
$$

which implies $\kappa_{\alpha}=44$ according to (8.12). For the Ag|aqueous electrolyte interface a similar value was used in [31]. 

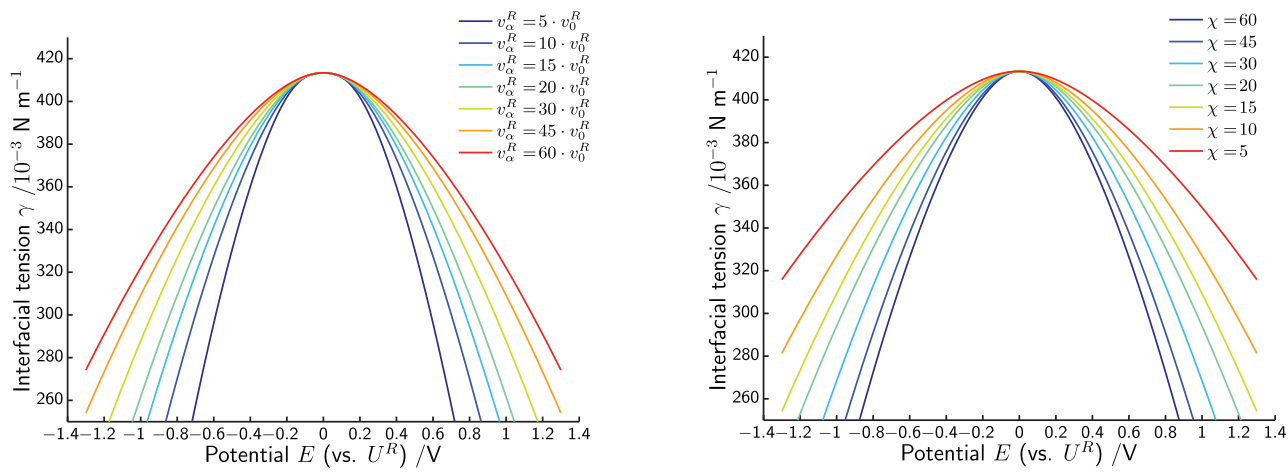

FiguRE 4. Parameter study of a monovalent, completely dissociated salt AC with bulk concentration $0.1 \mathrm{M}$. (a) Dependency of the interfacial tension $\gamma$ on the ionic specific volume $v_{\alpha}^{\text {ref }}$ with $\chi=15$. (b) Influence of the susceptibility $\chi$ on the interfacial tension $\gamma$ with $v_{\alpha}^{r e f}=45 \cdot v_{0}^{\text {ref }}$.

\subsubsection{Electric susceptibility}

The electric susceptibility $\chi$ can in general spatially depend on the local electrolyte concentration. It is usually supposed that $\chi$ gets smaller in the boundary layers where the solution gets more concentrated, cf. [7]. Non-constant susceptibility can be self-consistently modeled, cf. [41], but for simplicity we assume a constant $\chi$. Since the capacity maximum is essentially determined by the stored charge after saturation of the boundary layer sets in, it seems reasonable to approximate $\chi$ by a value considerably lower than the bulk value of $\chi \approx 80$ for pure water. Figure $4 \mathrm{~b}$ shows that variation of $\chi\left(v_{\alpha}^{r e f}\right.$ and $\kappa_{\alpha}$ according to (8.12) and (8.13), respectively) has similar effect on the surface tension like variation of $v_{\alpha}^{r e f}$ when $\chi$ is fixed. Thus, only the combination of both parameters can be fitted to experiments. Given the choice (8.13), we proceed in the following with

$$
\chi=15
$$

\subsubsection{Salt concentration}

Now that all necessary fit parameters for non-adsorbing monovalent salts are fixed by (8.11)-(8.14), we can study the dependency of the electrocapillarity curves on the salt concentration. It is well known that the interfacial tension dependents on the electrolyte concentration [22-24]. In Figure 5, we see that the numerical solutions of our model reproduce measurements quite well for a concentration range $0.0025-0.1 \mathrm{M}$ of a completely dissociated, non-adsorbing salt AC.

\subsection{Adsorption and reactions}

Specific adsorption of ions leads to a non-vanishing contribution $\gamma^{\mathrm{E}}$ which lowers the interfacial tension $\gamma$. While anions mostly adsorb in the right side of an electrocapillary diagram where more positive voltages are applied, the cations adsorb stronger for more 

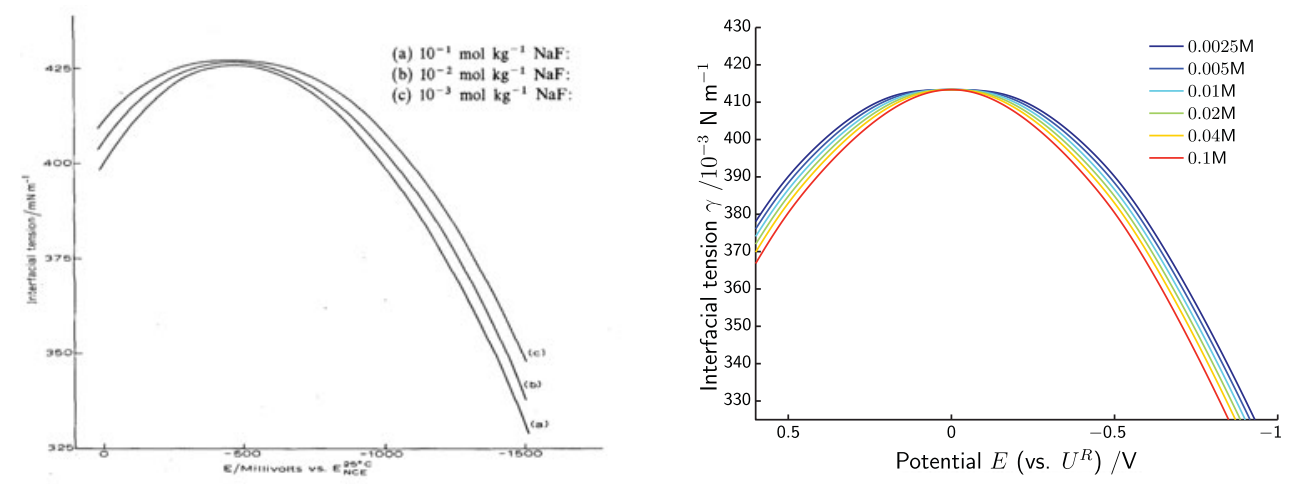

FIGURE 5. Measurement and simulation for aqueous solutions of non-adsorbing monovalent salts show good agreement for different salt concentrations. (a) Electrocapillarity curves for NaF solutions (based on Figure 2 of [12]). (b) Parameter study of the salt concentration for a monovalent salt AC.

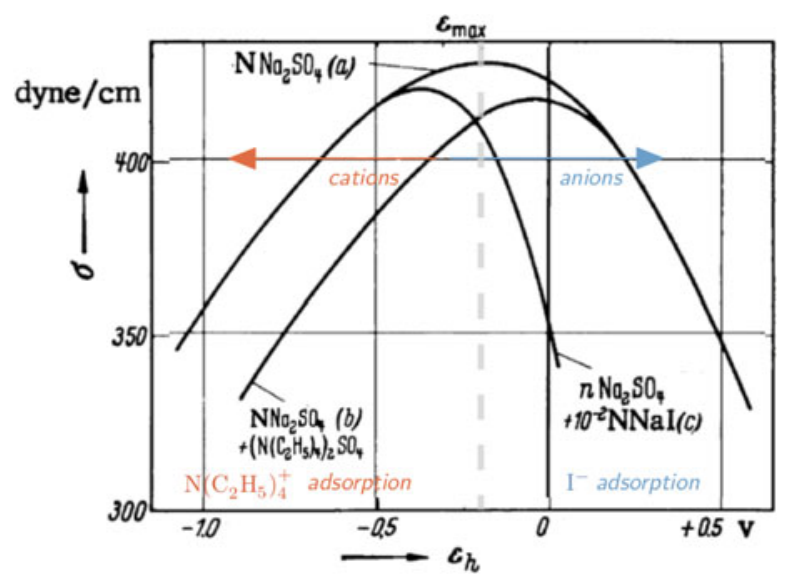

FIGURE 6. Measurement of the $\mathrm{Hg} \mid 0.05 \mathrm{M} \mathrm{Na}_{2} \mathrm{SO}_{4}$ interface (a) and with cation adsorbing additives (b) and anion adsorbing additives (c) (based on Figure 36 of [43]).

negative voltages. This is accompanied by a corresponding shift of the potential of zero charge in negative direction when anions adsorb, while for cations the is shift is in the positive direction, see Figure 6 for experimental data. The effect of the adsorption on the electrocapillary curves is controlled by the parameters $\Delta g_{\alpha}$ and $a_{\alpha}^{\text {ref }}$ studied below.

\subsubsection{Adsorption energies}

As a representative example for salts having the same non-adsorbing cation but different monovalent anions, e.g., $\mathrm{KCl}, \mathrm{KI}, \mathrm{KOH}$, we study the effect of varying the parameter $\Delta g_{\mathrm{A}^{-}}$on the computed electrocapillary curves of a $0.1 \mathrm{M}$ AC mixture. Since Figure 1a suggests that cations do not adsorb on $\mathrm{Hg}$, we set $\Delta g_{\mathrm{C}^{+}}=1 \mathrm{eV}$ according to (8.11). From Figure $7 \mathrm{a}$, we see that if we decrease the adsorption energy $\Delta g_{\mathrm{A}^{-}}$, the interfacial tension $\gamma$ 

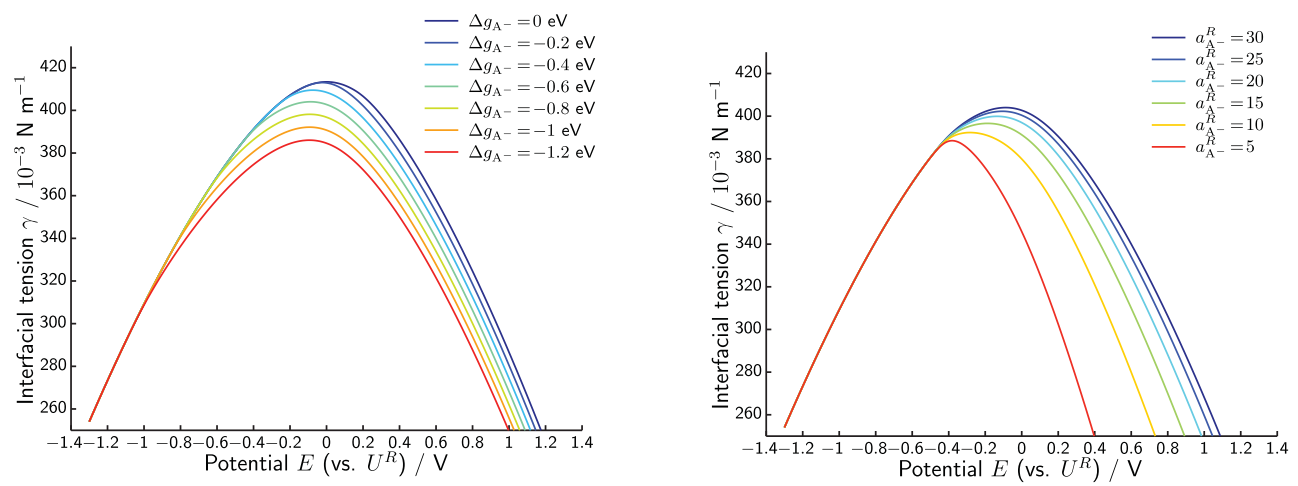

FiguRE 7. Parameter study of a completely dissociated salt $\mathrm{AC}$ of $0.1 \mathrm{M}$ concentration with an adsorbing anion $\mathrm{A}^{-}$. (a) Variation of the anion adsorption energy $\Delta g_{\mathrm{A}^{-}}$with $a_{\mathrm{A}^{-}}=30 a_{0}^{\text {ref }}$. (b) Variation of the anion specific area $a_{\mathrm{A}^{-}}$with adsorption energy $\Delta g_{\mathrm{A}^{-}}=-0.6 \mathrm{eV}$.

is lowered for larger applied potentials. Additionally, we observe that the position of the electrocapillary maximum moves to the left when the adsorption energy is decreased. Once the surface is completely covered by adsorbed anions, the double layer charge density does not depend on the parameter choice for $\Delta g_{\mathrm{A}^{-}}$any more. Thus, there is no visible difference in the slope of the curves in Figure 7a when the applied potential is large.

\subsubsection{Specific area}

Quite similar to the specific volume $v_{\alpha}^{r e f}$, the specific area $a_{\alpha}^{r e f}$ controls the maximal charge of adsorbed species that can be stored on the surface. For the ionic constituent on the surface we choose a simple relation between $a_{\alpha}^{\text {ref }}$ and $\kappa_{\alpha}$ analogous to (8.12), i.e.,

$$
a_{\alpha}^{r e f}=\left(1+\kappa_{\alpha}\right) a_{\mathrm{H}_{2} \mathrm{O}}^{r e f} .
$$

We assume that the cation $\mathrm{C}^{+}$does effectively not adsorb and set $\Delta g_{\mathrm{C}^{+}}=1 \mathrm{eV}$ and $\Delta g_{\mathrm{A}^{-}}=-0.6 \mathrm{eV}$. When the parameter $a_{\alpha}^{\text {ref }}$ then is varied in multiples of the solvent specific area $a_{\mathrm{H}_{2} \mathrm{O}}^{\text {ref }}$, we observe in Figure $7 \mathrm{~b}$ that anions can already adsorb more easily for lower values of $a_{\mathrm{A}^{-}}^{\text {ref }}$, resulting in a considerably larger curvature at the electrocapillarity maximum. Moreover, with decreasing specific area, we see a shift of the position of the maximum to the left. This shift appears stronger than that observed when decreasing $\Delta g_{\mathrm{A}^{-}}$in Figure $7 \mathrm{a}$. When the applied voltage is large, the slopes are again the same for all curves.

\subsubsection{Side remark on dissociation}

Due to the dissociation reaction $\mathrm{H}_{2} \mathrm{O} \rightleftharpoons \mathrm{H}^{+}+\mathrm{OH}^{-}$of water, there is always some - but possibly very small - amount of $\mathrm{H}^{+}$and $\mathrm{OH}^{-}$present in the electrolyte. It is reasonable to assume a similar dissociation process also on the surface, leading to the presence of adsorbed $\mathrm{H}^{+}$and $\mathrm{OH}^{-}$on the surface. Because $\mathrm{Hg}$ is usually considered not catalysing 
the self-ionization of water. We assume that for pure water the potential of zero charge is equal to zero. As a consequence, if $\mathrm{OH}^{-}$and $\mathrm{H}^{+}$adsorb on the metal surface, then their surface concentrations are equal at the potential of zero charge, i.e., $\Delta g_{\mathrm{OH}^{-}}=\Delta g_{\mathrm{H}^{+}}$. Then the reaction equilibrium conditions imply $\Delta g_{\mathrm{OH}^{-}}+\Delta g_{\mathrm{H}^{+}}=\Delta g_{\mathrm{H}_{2} \mathrm{O}}$ and thus

$$
\Delta g_{\mathrm{OH}^{-}}=\Delta g_{\mathrm{H}^{+}}=-0.0367 \mathrm{eV} \text {. }
$$

With these values, we find that the impact of adsorbed $\mathrm{H}^{+}$and $\mathrm{OH}^{-}$on the electrocapillarity curve is negligible. Note, however, that the actual surface concentration of $\mathrm{H}^{+}$and $\mathrm{OH}^{-}$is not zero.

\subsubsection{Electron transfer and metal surface reactions}

The steep anodic branches of several salts in the electrocapillarity curves of Figure 1 indicate the occurrence of either an electron transfer reaction

$$
\mathrm{A}^{-}-\mathrm{e}^{-} \rightleftharpoons \mathrm{A}
$$

or a reaction of the form

$$
2 \mathrm{Hg}^{2+}+2 \mathrm{~A}^{-}+2 \mathrm{e}^{-} \leftrightharpoons \mathrm{Hg}_{2} \mathrm{~A}_{2}
$$

like, e.g., mentioned by Frumkin [18]. We discuss the impact of both reactions, starting with the electron transfer $(8.17 a)$.

Consider a $0.1 \mathrm{M}$ AC solution where the anion may adsorb on the surface. Whenever $\Delta g_{\mathrm{A}} \ll \Delta g_{\mathrm{A}^{-}}$, the adsorbed anions will directly undergo the electron transfer reaction leaving almost no $\mathrm{A}^{-}$on the surface. If the reaction energy $\Delta g_{\mathrm{A}}$ is considerably larger than $\Delta g_{\mathrm{A}^{-}}$, the adsorbed anions will cause some visible decrease of the surface tension before the reaction sets in. Figure 8a displays a variation of $\Delta g_{\mathrm{A}}$. Because the reaction product $\mathrm{A}$ is uncharged, there is no solvation shell and thus the specific area $a_{\mathrm{A}}^{r e f}$ is comparable to $a_{\mathrm{H}_{2} \mathrm{O}}^{r e f}$. We set

$$
\kappa_{A}=0, \quad a_{A}^{r e f}=a_{\mathrm{H}_{2} \mathrm{O}}^{r e f} .
$$

The free solvent molecules are released to the volume according to the adsorption equilibrium condition leaving unoccupied sites on the surface which in turn allow for additional adsorption of $\mathrm{A}^{-}$from the volume. Because of the strongly different specific areas of $\mathrm{A}^{-}$and $\mathrm{A}$, we observe such a steep decrease of the electrocapillarity curve when the electron transfer reaction sets in.

Also the reaction $(8.17 b)$ produces a new species on the surface. We assume for the specific area $a_{\mathrm{Hg}_{2} \mathrm{~A}_{2}}^{r e f}$ the simple relation

$$
\kappa_{\mathrm{Hg}_{2} \mathrm{~A}_{2}}=0, \quad a_{\mathrm{Hg}_{2} \mathrm{~A}_{2}}^{r e f} \approx 2 a_{\mathrm{Hg}}^{r e f}+2 a_{\mathrm{A}}^{r e f} \approx 4 a_{\mathrm{H}_{2} \mathrm{O}}^{r e f} .
$$

Figure $8 \mathrm{~b}$ displays a variation of $\Delta g_{\mathrm{Hg}_{2} \mathrm{~A}_{2}}$, for a $0.1 \mathrm{M} \mathrm{AC}$ solution and $\Delta g_{\mathrm{A}^{-}}=-1 \mathrm{eV}$. The steep decrease originates from the rather small specific volume $a_{\mathrm{Hg}_{2} \mathrm{~A}_{2}}^{r e f}$ and the fact that there are two electrons involved in the reaction. 

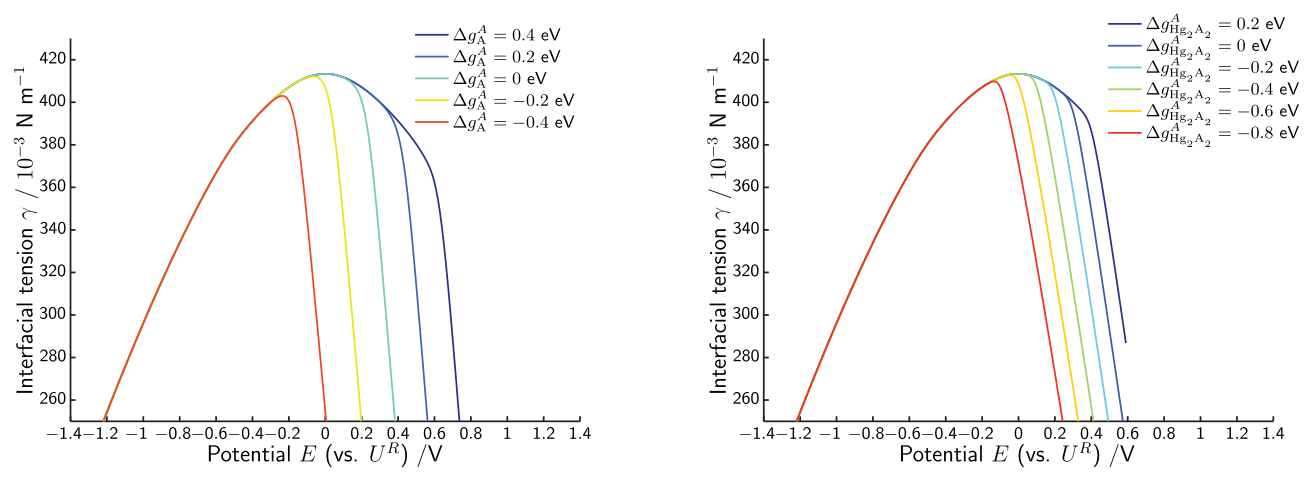

Figure 8. Parameter study of electron transfer and reactions with the metal. (a) Variation of the adsorption energy $\Delta g_{\mathrm{A}}$ related to the electron transfer reaction $\mathrm{A}^{-}-\mathrm{e}^{-} \rightleftharpoons \mathrm{A}+\kappa_{\mathrm{A}^{-}} \cdot \mathrm{H}_{2} \mathrm{O}$. (b) Variation of $\Delta g_{\mathrm{Hg} 2} A_{2}$ related to the reaction $2 \mathrm{Hg}^{2+}+2 \mathrm{~A}^{-}+2 \mathrm{e}^{-} \rightleftharpoons \mathrm{Hg}_{2} \mathrm{~A}_{2}+2 \kappa_{\mathrm{A}^{-}} \mathrm{H}_{2} \mathrm{O}$.

\subsection{Calculated electrocapilarity curves for Figure 1a}

Based on the parameter study above, we can now specify model parameters that allow reproduction of the introductory electrocapillarity curves Figure 1b. Then, we discuss quantitative and qualitative agreement of our results.

\subsubsection{Parameter choice}

We consider the monovalent salts $\mathrm{KOH}, \mathrm{NaCl}, \mathrm{NaBr}, \mathrm{KI}, \mathrm{KCNS}$, and $\mathrm{Ca}\left(\mathrm{NO}_{3}\right)_{2} \cdot{ }^{2}$ The goal is to reproduce the height and position of the electrocapillarity maximum as well as the slopes for large positive applied voltages and the endpoints of the curves.

For $\mathrm{CaNO}_{3}^{+}$and the alkali cations we set $\Delta g_{\mathrm{C}^{+}}=1 \mathrm{eV}$ according to (8.11), whereby effectively no adsorption occurs. The adsorption energy of $\mathrm{H}^{+}$is given as $\Delta g_{\mathrm{H}^{+}}=$ $-0.0367 \mathrm{eV}$ according to (8.16). With the assumption of equal specific volume for $\mathrm{Na}^{+}$, $\mathrm{K}^{+}$and $\mathrm{CaNO}_{3}^{+}$the left electrocapillarity arc's coincide in all cases.

All anions are assumed to adsorb on $\mathrm{Hg}$ and strip of a part of their salvation shell. Among the considered halogenes, this process is strongest for iodide, followed by bromide and chloride, we choose

$$
\Delta g_{\mathrm{I}^{-}}=-0.6 \mathrm{eV}, \quad \Delta g_{\mathrm{Br}^{-}}=-0.4 \mathrm{eV}, \quad \text { and } \quad \Delta g_{\mathrm{Cl}^{-}}=-0.2 \mathrm{eV}
$$

The anions $\mathrm{CNS}^{-}, \mathrm{CaNO}^{-}$, and $\mathrm{OH}^{-}$may also adsorb on $\mathrm{Hg}$, where we have chosen

$$
\Delta g_{\mathrm{CNS}^{-}}=-0.45 \mathrm{eV} \text { and } \Delta g_{\mathrm{NO}^{-}}=-0.35 \mathrm{eV}
$$

and $\Delta g_{\mathrm{OH}^{-}}=-0.0367 \mathrm{eV}$ according to (8.16). It seems likely that stronger adsorbed constituents also have a smaller solvation shell (and thus a specific area), whereby we

2 Note that we assume here actually a complete (or major) dissociation of $\mathrm{Ca}\left(\mathrm{NO}_{3}\right)_{2}$ in $\mathrm{CaNO}_{3}^{+}$ and $\mathrm{NO}_{3}^{-}$, similar as $\mathrm{H}_{2} \mathrm{SO}_{4}$ dissociates mainly into $\mathrm{HSO}_{4}^{-}$and $\mathrm{H}^{+}$. A further dissociation of $\mathrm{CaNO}_{3}^{+}$into $\mathrm{Ca}^{2+}$ and $\mathrm{NO}_{3}^{-}$is possible within our framework, but requires some more discussion of parameters for the multivalent ion $\mathrm{Ca}^{2+}$, i.e., its specific volume. 
choose

$$
a_{\mathrm{I}^{-}}^{r e f}=10 a_{\mathrm{H}_{2} \mathrm{O}}^{r e f}, \quad a_{\mathrm{Br}^{-}}^{r e f}=15 a_{\mathrm{H}_{2} \mathrm{O}}^{r e f}, \quad \text { and } \quad a_{\mathrm{Cl}^{-}}^{r e f}=20 a_{\mathrm{H}_{2} \mathrm{O}}^{r e f}
$$

With respect to the anions $\mathrm{CNS}^{-}, \mathrm{CaNO}^{-}$, and $\mathrm{OH}^{-}$, we choose specific areas

$$
a_{\mathrm{CNS}^{-}}^{r e f}=10 a_{\mathrm{H}_{2} \mathrm{O}}^{r e f} \quad a_{\mathrm{CaNO} 3^{-}}^{r e f}=35 a_{\mathrm{H}_{2} \mathrm{O}}^{r e f} \quad \text { and } \quad a_{\mathrm{OH}^{-}}^{r e f}=30 a_{\mathrm{H}_{2} \mathrm{O}}^{r e f}
$$

We consider further the reactions of the type (8.17b) between $\mathrm{Hg}$ and the anions $\mathrm{I}^{-}, \mathrm{Br}^{-}, \mathrm{Cl}^{-}$and $\mathrm{OH}^{-}$with the energies

$$
\begin{aligned}
\Delta g_{\mathrm{Hg}_{2} \mathrm{I}_{2}} & =-0.8 \mathrm{eV}, \quad \Delta g_{\mathrm{Hg}_{2} \mathrm{Br}_{2}}=-0.4 \mathrm{eV}, \\
\Delta g_{\mathrm{Hg}_{2} \mathrm{Cl}_{2}} & =-0.1 \mathrm{eV}, \quad \Delta g_{\mathrm{Hg}_{2}(\mathrm{OH})_{2}}=-0.1 \mathrm{eV} .
\end{aligned}
$$

The specific areas of the adsorbed, uncharged reaction products are chosen according (8.19) as $a_{\beta}^{r e f}=4 a_{\mathrm{H}_{2} \mathrm{O}}^{r e f}$.

\subsubsection{Discussion on the calculated electrocapilarity curves}

With this set of parameters, we compute the electrocapillarity curves of the respective $0.1 \mathrm{M}$ salt solutions in the potential range from $-1.3 \mathrm{~V}$ to $0.6 \mathrm{~V}$ and obtain a very good qualitative and quantitative agreement to the experimental data of Grahame and Gouy (cf. Figure 1). It is to emphasize that our results are based on the rather simple material functions given in Section 7.1. Near to the end points of the experimental curves it is likely that there are additional phenomena, which are not included in our calculations so far. For example, $\mathrm{Hg}$ might start dissolving into solution or there can be reactions different from the considered net reactions (4.19). If some adsorbed ions undergo a charge transfer reaction and the reaction product can desorb back to the solution, e.g., like $2 \mathrm{H}^{+}+2 \mathrm{e}^{-} \rightleftharpoons \mathrm{H}_{2}$ or $2 \mathrm{Cl}^{-}-2 \mathrm{e}^{-} \rightleftharpoons \mathrm{Cl}_{2}$, then a net charge transfer occurs which invalidates the current-charge relation (7.17). While all these phenomena con be an origin of deviation between experimental and computed data, it is remarkable, that our computed electrocapilarity curves reproduce the experimental curves well within such a wide potential range.

Seemingly, there is some qualitative difference between the measured data and the computed electrocapillarity curves which show a tiny kink when the reaction $(8.17 b)$ sets in (cf. Figure $1 \mathrm{~b}$ at $0.1 \mathrm{~V}$ for $\mathrm{I}^{-}, 0.25 \mathrm{~V}$ for $\mathrm{Br}^{-}$and $0.4 \mathrm{~V}$ for $\mathrm{Cl}^{-}$). But one has to keep in mind that although the continuous plots of the electrocapillarity curves in Figure 1a suggest an infinite precision of the measurement with respect to the applied potential, they are in fact based on discrete data points. In fact, the data sets of Gouy are actually based on a very coarse potential discretization of $0.1 \mathrm{~V}$. If we follow this procedure, i.e., interpolate the computed data points corresponding to a coarse discretization of $0.1 \mathrm{~V}$ for the applied potential $E$, this kink is not visible any more (cf. Figure 9). 


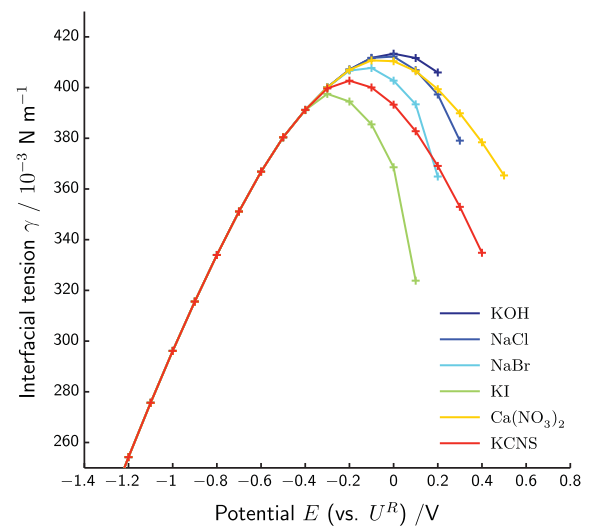

FIgURE 9. Computation of the interfacial tension based on our model with the parameters $(8.20 a)-(8.20 e)$ and a voltage discretization of $0.1 \mathrm{~V}$.

Due to the constant potential difference $U^{\mathrm{M}}$ and the simple relation (8.2) between the potential difference $U^{\mathrm{E}}$ and measured potential $E$, the Lippmann equation (4.18) can be rewritten as

$$
\frac{d \gamma}{d E}=-Q
$$

This shows that the electrocapillarity maximum indeed corresponds to the potential of zero charge $E^{0}$, i.e.

$$
\left.\frac{d \gamma}{d E}\right|_{E=E^{0}}=\left.0 \quad \Leftrightarrow \quad Q\right|_{E=E^{0}}=0
$$

\subsection{Capacity curves}

The double layer capacity $C$ is related to the double layer charge density $Q$ according to $C=-d Q / d E$, cf. $[8,37,38]$. From the representation formulas of Section 7.3 , we have $Q_{\mathrm{BL}}$ and $\underset{s}{Q}$ given as functions of $U^{\mathrm{E}}$ and $\gamma_{s}^{\mathrm{E}}$. Thus, by differentiation of (7.25) and (7.26) it is possible to derive an algebraic system that directly determines $C$ in dependence of $E$. The actual functional representations of $C$ are derived in detail in [31]. From the Lippmann equation (8.21), we obtain the relation between interfacial tension $\gamma$ and differential capacity $C$ as

$$
-\frac{d \gamma^{2}}{d^{2} E}=C
$$

A computation of the double layer capacity based on our model and the parameters $(8.20 a)-(8.20 e)$ for the various $\mathrm{Hg} \mid$ aqueous salt solution interfaces is given in Figure 10 . Coming from negative potentials we first observe a local capacity maximum that is the same for all considered interfaces and is mainly due to storage of the boundary layer charge density $Q_{\mathrm{BL}}^{\mathrm{E}}$. Proceeding in direction towards more positive potentials, each curve 


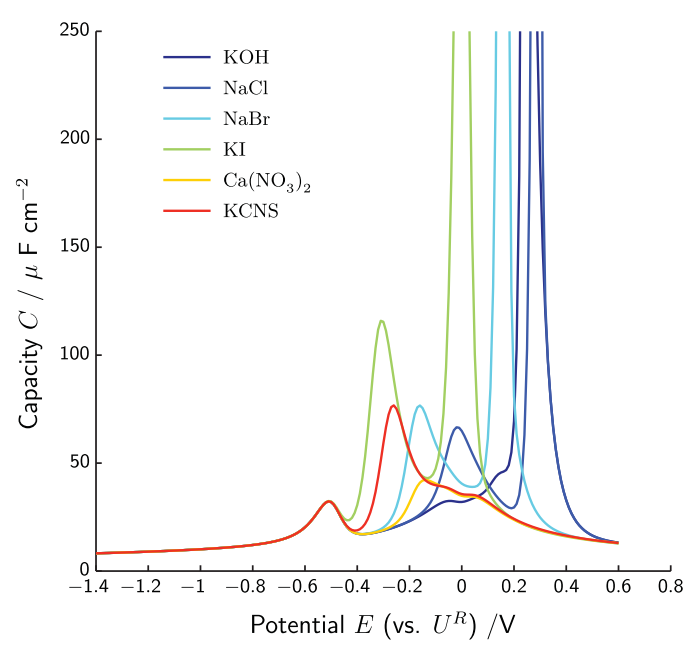

FIGURE 10. Double layer capacity $C(E)$ based on our model with parameters $(8.20 a)-(8.20 e)$.

shows a local minimum at the respective potential of zero charge, but the position of the minima differ between the individual curves. Next, we observe a local capacity maximum significantly higher than the first one (except for $\mathrm{KOH}$ ), where position and height differ between the curves. This second local maximum has to be attributed to the capacity related to the surface charge density $Q_{s}^{\mathrm{E}}$. While there also is a boundary layer contribution to the capacity for potential positive w.r.t. the potential of zero charge, it is effectively hidden under the dominant surface part of $C$. Finally, we observe very pronounced capacity peaks which are related to the onset of the reaction $(8.17 b)$.

\section{Summary}

\subsection{General setting}

For continuum models of electrochemical interfaces in the context of thermodynamics, the necessary requirements are to be compatible with balance equations of mass, momentum and energy and the 2nd law of thermodynamics in the sense that entropy production is guaranteed to be non-negative. No less important is the compatibility with experimentally well confirmed fundamental equations of electrochemistry like the Lippmann equation. In this paper, we showed how the Lippmann equation can be derived - or recovered - from the continuum thermodynamic model within an asymptotic regime that is relevant for macroscopic measurements. Thereby, we clarified the role of the Lippmann equation as an implication of the model equations in the bulk domains and on the surface rather than being an independent axiom. Since no information about the material specific free energy densities was required, the role of the Lippmann equation as an universal identity is confirmed as far as it is applied in a sufficiently macroscopic setting. Moreover, we are able to give sharp definitions of all quantities appearing in the equation. 
In particular, our derivation reveals that the boundary layer contributions $\widetilde{\gamma}^{ \pm}$to the interfacial tension $\gamma$ are always non-negative and can only reduce the interfacial tension. Moreover, we see that by measuring interface charge it is only possible to draw conclusions about the combined interfacial tension $\gamma$, but not about the thermodynamic surface tension $\gamma$.

\subsection{Validity and applicability}

The asymptotic framework used in the derivation relies on scaling relations of the dimensionless parameters which are implied by the chosen reference quantities like length scales and number densities. Thereby, it allows to estimate the applicability of the Lippmann equation in a specific parameter range. In particular, the minimal curvature radius of the interface has to be larger than the Debye length by about one order of magnitude, i.e.,

$$
\lambda L^{r e f} k_{M} \ll 1 \text {. }
$$

Since we derived the Lippmann equation without any assumption of the material specific behaviour encoded in the free energy densities, the derivation is also valid for general liquid-liquid interfaces. Nevertheless, further modelling is necessary to specify a suitable free energy for such an liquid-liquid interface before it is possible to actually calculate all contributions to the double layer charge density and the interfacial tension.

\subsection{Liquid metal-aqueous electrolyte interface}

For the example of the liquid metal-aqueous electrolyte interface an explicit material model consisting of free energy densities can be applied. This enables us to derive representation equations in terms of the applied potential $E$ for all surface quantities and the layer charge density and layer tension and thereby allows the efficient numerical computation of electrocapilarity curves. Detailed investigations on the various equilibrium parameters of our model were carried out in order to provide insight on the respective dependency. This finally allows to identify a parameter set such that it is possible to reproduce experimental electrocapilarity curves with a remarkable qualitative and quantitative agreement in a $2 \mathrm{~V}$ potential range.

It is worth to point out that the definition of the double layer capacity defined by the Lippmann equation is consistent with the definition of the differential capacity in [31]. We see that adsorption and charging of the double layer always results in a reduction of the thermodynamic surface tension $\gamma$, in addition to the reduction of the boundary layer tension $\widetilde{\gamma}^{ \pm}$by charge accumulation in the boundary layers. Together with the monotonicity properties of the representation equations this leads to the general parabolic shape of the electrocapilarity curves.

In the literature, there has been some discussion about the electrode charge and the applicability of the Lippmann equation - or generalizations thereof - in the presence of Faradayic currents or for the case of a reversible electrode, cf. [19,20]. The general derivation of the Lippmann equation given above does not require the assumption of a perfectly polarizable electrode but stays also valid in the presence of Fradayic currents. 
Only one has to keep in mind that the charge-current relation that can be used to measure the double layer charge by an experiment independent from the Lippmann equation, was based on the assumption that there is no charge transfer across the interface.

\section{References}

[1] Adamson, A. W. \& Gast, A. P. (1997) Physical Chemistry of Surfaces, 6st ed., John Wiley \& Sons, New York.

[2] Albano, A. M. \& Bedeaux, D. (1987) Non-equilibrium electro-thermodynamics of polarizable multicomponent fluids with an interface. Physica A 147(1-2), 407-435.

[3] BARD, A. J. \& FAulKner, L. R. (2001) Electrochemical Methods: Fundamentals and Applications, 2nd ed., Wiley, New York.

[4] Bazant, M. Z., Thornton, K. \& Ajdari, A. (2004) Diffuse-charge dynamics in electrochemical systems. Phys. Rev. E 70, 021506, .

[5] Bazant, M. Z., Chu, K. T. \& Bayly, B. J. (2005) Current-voltage relations for electrochemical thin films. SIAM J. Appl. Math. 65(5), 1463-1484.

[6] Bedeaux, D. (1986) Nonequilibrium thermodynamics and statistical physics of surfaces. In: Prigogine Ilya \& S. A. Rice (editors), Advances in Chemical Physics, Vol. 64, John Wiley \& Sons, New York, pp. 47-109.

[7] Bockris, J. O. M., Devanathan, M. A. V. \& Muller K. (1963) On the structure of charged interfaces. Proc. Roy. Soc. Lond. A 274(1356), 55-79.

[8] Bockris, J. O. M., Reddy, A. K. N. \& Gamboa-Aldeco, M. E. (2002) Modern Electrochemistry A: Fundamentals of Electrodics, 2nd ed., Vol. 2, Kluwer Academic Publishers, New York.

[9] Bothe, D. \& Dreyer, W. (2015) Continuum thermodynamics of chemically reacting fluid mixtures. Acta Mech. 226(6), 1757-1805.

[10] Caginalp, G. \& Fife, P. (1988) Dynamics of layered interfaces arising from phase boundaries. SIAM J. Appl. Math. 48(3), 506-518.

[11] Chernenko, A. A. (1963) Theory of the passage of a DC current through a solution of a binary electrolyte. Dokl. Akad. Nauk SSSR 153, 1129.

[12] Cuong, N. H. , D’Alkaine, C. V. , Jenard, A. \& Hurwitz, H. D. (1974) The surface phase at the ideal polarized mercury electrode: 2. coulostatic measurements at the $\mathrm{Hg}$ electrode in dilute $\mathrm{NaF}$ aqueous solutions at various temperatures. J. Electroanal. Chem. 51(2), 377 393.

[13] Defay, D. \& Sanfeld, A. (1967) The tensor of pressure in spherical and plane electrocapillary layers. Electrochim. Acta 12(8), 913-926.

[14] DeGroot, S. R. \& Mazur, P. (1984) Non-equilibrium Thermodynamics, Dover Publications Inc., New York.

[15] Dreyer, W., Giesselmann, J. \& Kraus, C. (2014) A compressible mixture model with phase transition. Physica D 273-274, 1-13.

[16] Dreyer, W., Guhlke, C. \& MÜLler R. (2015) Modeling of electrochemical double layers in thermodynamic non-equilibrium. Phys. Chem. Chem. Phys. 17, 27176-27194.

[17] Foster, J. M. , Snaith, H. J., Leijtens, T. \& Richardson, G. (2014) A model for the operation of perovskite based hybrid solar cells: Formulation, analysis, and comparison to experiment. SIAM J. Appl. Math. 74(6), 1935-1966.

[18] Frumkin, A.(1928) Die Elektrokapillarkurve. Ergebnisse der Exakten Naturwissenschaften. Springer, Berlin, Heidelberg, Chapter 7, pp. 235-275.

[19] Frumkin, A. N. (1968) The Lippmann equation. Comments on part XXII of the paper "On the impedance of galvanic cells", by Timmer, Sluyters-Rehbach and Sluyters. J. Electroanal. Chem. 18(3), 328-329.

[20] Frumkin, A., Petry, O. \& Damaskin, B. (1979) The notion of the electrode charge and the Lippmann equation. J. Electroanal. Chem. 27(1), 81-100. 
[21] Funrmann, J. (2015) Comparison and numerical treatment of generalised Nernst-Planck models. Comp. Phys. Comm. 196(Supplement C), 166-178.

[22] Gouy, L. G. (1903) Sur la fonction électrocapillaire. Ann. Chim. Phys. 29, 145-241.

[23] Gouy, L. G. (1906) Sur la fonction électrocapillaire. Ann. Chim. Phys. 8, 291-363.

[24] Gouy, L. G. (1906) Sur la fonction électrocapillaire. Ann. Chim. Phys. 9, 75-139.

[25] Gouy, L. G. (1910) Sur la constitution de la charge électrique à la surface d'un électrolyte. $J$. Phys. Théor. Appl. 9(4), 457-468.

[26] Grafov, B. M. \& Chernenko, A. A. (1962) Theory of direct-current flow through a binary electrolyte solution. Dokl. Akad. Nauk SSSR 146, 135.

[27] Grahame, D. C. (1947) The electrical double layer and the theory of electrocapillarity. Chem. Rev. 41(3), 441-501.

[28] Guhlke, C. (2015) Theorie Der Elektrochemischen Grenzfäche, $\mathrm{PhD}$ thesis, TU-Berlin, Germany.

[29] Hurwitz, H. D. \& D'Alkaine, C. V. (1973) The surface phase at the ideal polarized mercury electrode: I. General thermodynamic and hydrostatic considerations of ionic adsorption. $J$. Electroanal. Chem. 42(1), 77-103.

[30] Jasper, J. J. (1972) The surface tension of pure liquid compounds. J. Phys. Chem. Ref. Data 1(4), 841-1010.

[31] Landstorfer, M., Guhlke, C. \& Dreyer, W.(2016) Theory and structure of the metalelectrolyte interface incorporating adsorption and solvation effects. Electrochimica Acta 201, 187-219.

[32] Lippmann, G. (1873) Beziehungen zwischen den capillaren und elektrischen Erscheinungen. Ann. Phys. 225(8), 546-561.

[33] Lippmann, G.(1875) Relations Entre les Phénomènes électriques et Capillaires, Ann. Chim. Phys. 5(11), 494-549.

[34] MeIXner, J. \& ReIK, H. G. (1959) Thermodynamik der Irreversiblen Prozesse, Vol. 3, Springer, Berlin, pp. 413-523.

[35] MüLler, I. (1985) Thermodynamics, Interaction of Mechanics and Mathematics Series. Pitman Advanced Publishing Program, Boston.

[36] Newman, J. (1965) The polarized diffuse double layer. Trans. Faraday Soc. 61, 2229-2237.

[37] Newman, J. \& Thomas-Alyea, K. E. (2004) Electrochemical Systems. John Wiley \& Sons, Hoboken, New Jersey.

[38] Nic, M., Jirat, J. \& Kosata B. (2012) IUPAC compendium of chemical terminology (gold book) [online]. URL: goldbook.iupac.org/index.html. Release 2.3.3b, 2017/03/27.

[39] Pego, R. L. (1989) Front migration in the nonlinear Cahn-Hilliard equation. Proc. R. Soc. Lond. A 422(1863), 261-278.

[40] Richardson, G. (2000) A multiscale approach to modelling electrochemical processes occurring across the cell membrane with application to transmission of action potentials. Math. Med. Biol. 26(3), 201-224.

[41] SANFeld, A. (1968) Introduction to the Thermodynamics of Charged and Polarized Layers, Vol. 10 of Monographs in Statistical Physics and Thermodynamics. John Wiley \& Sons, London, New York, Sydney.

[42] Schmeiser, C. \& Unterreiter, A. (1994) The derivation of analytic device models by asymptotic methods. In: W. M. Coughran, J. Cole, P. Lloyd \& J. K. White (editors), Semiconductors: Part II, Springer, New York, pp. 343-363.

[43] Vetter, K. J. (1967) Electrochemical Kinetics, Academic Press, New York.

\section{Appendix A Relation between $U^{\mathrm{E}}$ and the measured cell potential $E$}

Let us consider a experimental setup, where the metal and the reference electrode $\mathrm{R}$ are connected via cables $\mathrm{C}_{1}$ and $\mathrm{C}_{2}$ to a voltmeter $\mathrm{V}$ which measures a voltage $E$ between its two identical, metallic plates $\mathrm{V}_{1}$ and $\mathrm{V}_{2}$. The electrochemical cell, including measuring 
device and cables, may thus be written as

$$
\underbrace{\mathrm{V}_{1}\left|\mathrm{C}_{1}\right| \mathrm{M}|\mathrm{E}| \mathrm{R}\left|\mathrm{C}_{2}\right| \mathrm{V}_{2}}_{E=\varphi_{\mathrm{V}_{1}}-\varphi_{\mathrm{S}}}
$$

The measured cell potential $E$ then corresponds to the surface potential difference between the two plates of the voltmeter, i.e.,

$$
E=\underset{s}{\varphi_{\mathrm{V}_{1}}}-\varphi_{S}
$$

Due to the continuity of electrochemical potential $\mu_{e}-e_{0} \varphi$ of the electrons in the different metals and at metal contacts, we have

$$
E=U^{\mathrm{E}}+U^{\mathrm{R}} \quad \text { with } \quad U^{\mathrm{R}}=-\frac{1}{e_{0}}\left(\mu_{s}^{\mathrm{M}}-\mu_{s}^{\mathrm{R}}\right)-U^{\mathrm{R}, \mathrm{E}}
$$

where $U^{\mathrm{R}, \mathrm{E}}=\varphi^{\mathrm{R}}-\varphi^{\mathrm{E}}$ denotes the potential difference between bulk electrolyte and surface potential of the reference electrode. We follow the common assumption that the reference potential $U^{\mathrm{R}, \mathrm{E}}=$ const. is constant [8]. If we assume for $\mathrm{R}$ an analogous material model to the one used for $\mathrm{M}$, then $\mu_{s}^{\mathrm{R}}$ and thus also $U^{\mathrm{R}}$ is a constant.

\section{Appendix B Current-charge relation of the metal/electrolyte interface}

The derivation of the relation (7.17) between the current and the electric charge is based on non-equilibrium thermodynamics $[14,35]$. First of all, we introduce the domains of metallic and electrolytic boundary layer $^{3}$ at the metal/electrolyte interface $S$, viz., $\Omega_{\mathrm{BL}}^{\mathrm{M}}:=\left\{\boldsymbol{x}+\varepsilon \boldsymbol{v} \mid x \in S,, 0<\varepsilon<\varepsilon_{0}\right\} \subset \Omega^{\mathrm{M}}$ and $\Omega_{\mathrm{BL}}^{\mathrm{E}}:=\left\{\boldsymbol{x}-\varepsilon \boldsymbol{v} \mid x \in S,, 0<\varepsilon<\varepsilon_{0}\right\} \subset \Omega^{\mathrm{E}}$.

The electric current $I\left[\mathrm{~A} / \mathrm{m}^{2}\right]$ flowing into the double layer through the surface $A^{\mathrm{M}}$ between $\Omega_{\mathrm{BL}}^{\mathrm{M}}$ and $\Omega^{\mathrm{M}} \backslash \Omega_{\mathrm{BL}}^{\mathrm{M}}$ is given by

$$
I=-\frac{1}{\left|A^{\mathrm{M}}\right|} \int_{A^{\mathrm{M}}}\left(\frac{z_{e} e_{0}}{m_{e}} \boldsymbol{j}_{e} \cdot \boldsymbol{v}+\frac{z_{M} e_{0}}{m_{M}} \boldsymbol{j}_{M} \cdot \boldsymbol{v}\right) d a .
$$

Herein, $\boldsymbol{j}_{e, M}\left[\mathrm{~kg} / \mathrm{s} \mathrm{m}^{2}\right]$ are the mass flux densities of electrons and metal ions, respectively. The following objective is the representation of the right hand side of (B 1) by time derivatives. To this purpose, we introduce the mass balance equations for electrons and metal ions for $\Omega_{\mathrm{BL}}^{\mathrm{M}}$,

$$
\frac{d}{d t} \int_{\Omega_{\mathrm{BL}}^{\mathrm{M}}} m_{\alpha} n_{\alpha} d x=-\int_{A^{\mathrm{M}}} \boldsymbol{j}_{\alpha} \cdot \boldsymbol{v} d a+\int_{S} \boldsymbol{j}_{\alpha} \cdot \boldsymbol{v} d a, \quad \alpha=e, M
$$

Note that the normal vector at $A^{\mathrm{M}}$ points outward $\Omega_{\mathrm{BL}}^{\mathrm{M}}$ and at $S$ points into $\Omega_{\mathrm{BL}}^{\mathrm{M}}$. Next,

${ }^{3}$ For simplicity, we assume here that $S$ is a closed surface in $\mathbb{R}^{3}$, to avoid discussion about the phenomena at the boundary lines $\partial S$ of $S$. By this, we have assumed that the charge transport over the contact lines $\partial S$ only has a minor impact on the total charge transport across the surface $S$. 
we introduce the corresponding surface balance equations, which are used to determine the fluxes at $S$ in (B 2),

$$
\frac{d}{d t} \int_{S}\left(m_{\alpha} n_{\alpha}\right) d a=\int_{S} r_{\alpha} d a-\int_{S} \boldsymbol{j}_{\alpha} \cdot \boldsymbol{v} d a, \quad \alpha=e, M
$$

Herein denotes $r_{\alpha}$ the production density of electrons and metal ions due to surface reactions on $S$. According to the surface reaction (4.19) the production densities are defined by

$$
r_{\alpha}=-\sum_{\beta \in \mathcal{M}_{S} \backslash M_{\mathrm{E}, M}} v_{\alpha \beta} m_{\alpha} R^{\beta}, \quad \alpha=e, M .
$$

Here, $R^{\beta}$ denotes the reaction rates of the net reaction of the surface constituent $A_{\beta}$, $\beta \in \mathcal{M}_{S} \backslash \mathcal{M}_{\mathrm{E}, \mathrm{M}}$. For the exclusive surface constituents $A_{\beta}$, where no corresponding bulk species exists, the surface mass balances read

$$
\frac{d}{d t} \int_{S}\left(m_{\beta} n_{s}\right) d a=\int_{S} r_{\beta} d a, \quad \beta \in \mathcal{M}_{S} \backslash \mathcal{M}_{\mathrm{E}, \mathrm{M}} .
$$

According to (4.19) the production densities are related to the reaction rates by

$$
r_{\beta}=m_{\beta} R^{\beta}, \quad \beta \in \mathcal{M}_{S} \backslash \mathcal{M}_{\mathrm{E}, \mathrm{M}} .
$$

Inserting the balance equations (B 2), (B 3), (B 5) into (B 1) and using the relations (B 4) and (B 6) yields

$$
I=\frac{1}{\left|A^{\mathrm{M}}\right|} \frac{d}{d t}\left(\int_{\Omega_{\mathrm{BL}}^{\mathrm{M}}} n^{\mathrm{F}} d x+\int_{S} \sum_{\alpha \in \mathcal{M}_{\mathrm{M}}}\left(z_{\alpha} e_{0} n_{\alpha}\right) d a+\int_{S}\left(\sum_{\alpha \in \mathcal{M}_{\mathrm{M}}} \sum_{\beta \in \mathcal{M}_{S} \backslash M_{\mathrm{E}, \mathrm{M}}} z_{\alpha} e_{0} v_{\alpha \beta} n_{\beta}\right) d a\right) .
$$

We assume that the current itself is of first order, i.e., $I=\lambda I^{(0)}$, than the first non-vanishing order of (B 7) reads

$$
I=\frac{d}{d t}\left(\int_{0}^{+\infty} n^{\mathrm{F}} d x+\sum_{\alpha \in \mathcal{M}_{\mathcal{M}}}\left(z_{\alpha} e_{0} n_{\alpha}\right) d a+\left(\sum_{\alpha \in \mathcal{M}_{\mathcal{M}}} \sum_{\beta \in \mathcal{M}_{S} \backslash M_{\mathrm{E}, \mathcal{M}}} z_{\alpha} e_{0} v_{\alpha \beta} n_{\beta}\right) d a\right) .
$$

The charge conservation (2.3a) for reaction (4.19) reads $z_{\beta}=\sum_{\alpha \in \mathcal{M}_{\mathrm{E}, M}} v_{\alpha \beta} z_{\alpha}$ for all $\beta \in \mathcal{M}_{S} \backslash \mathcal{M}_{\mathrm{E}, \mathrm{M}}$. Together with the electroneutrality condition (4.6b) we get the sought relation (7.17). 